\title{
A Riemann Coupled Edge (RCE) 1D-2D finite volume inundation and solute transport model
}

\author{
M. Morales-Hernández, • A. Lacasta • J. \\ Murillo • P. Brufau • P. García-Navarro
}

Received: date / Accepted: date

\begin{abstract}
A novel 1D-2D shallow water model based on the resolution of the Riemann Problem at the coupled grid edges is presented in this work. Both the $1 \mathrm{D}$ and the $2 \mathrm{D}$ shallow water models are implemented in a finite volume framework using Approximate Roe's solvers that are able to deal correctly with wet/dry fronts. After an appropriate geometric link between the models, it is possible to define local Riemann Problems at each coupled interface and estimate the contributions that update the cell solutions from the interfaces. The solute transport equation is also incorporated into the proposed procedure. The numerical results achieved by the $1 \mathrm{D}-2 \mathrm{D}$ coupled model are compared against a complete $2 \mathrm{D}$ model, which is considered the reference solution. The computational time is also examined.
\end{abstract}

Keywords 1D-2D coupled model · shallow water $\cdot$ conservation · solute transport · Riemann Problem

\section{Introduction}

The simulation of flooding events is a common practice for companies and river basin administrations that nowadays demand more accurate and faster models involving larger temporal and spatial scales. One dimensional (1D) hydraulic models have been previously used for these purposes, mainly due to their efficiency. Nevertheless, they have been deprecated as a result of the intrinsic limitation in simulating the floodplain-river interactions and the associated loss of accuracy. On the other hand, two-dimensional (2D) hydraulic models

M. Morales-Hernández

Fluid Mechanics, Universidad Zaragoza, LIFTEC-CSIC, C/ María de Luna, 3 50018, Zaragoza

Tel.: +34876555316

Fax: +123-45-678910

E-mail: mmorales@unizar.es 
have been accepted as a way to overcome the $1 \mathrm{D}$ constraints. These models are able to simulate over complex geometries involving wet/dry boundaries. However, as they require a great level of detail in the form of number of cells to ensure a reliable accuracy, their disadvantage resides in the large amount of computations, which turns into unaffordable simulations for real-time systems or large space and time domains.

Therefore, coupled 1D-2D models naturally arise when combining the advantages of pure $1 \mathrm{D}$ and $2 \mathrm{D}$ hydrodynamical models. In fact, the implementation of a complete 1D-2D model seems to be a good solution to eliminate not only the limitations of the $1 \mathrm{D}$ model when modelling flooding waves over $2 \mathrm{D}$ domains, but also the uncertainty in the $2 \mathrm{D}$ model associated to the optimal discrete representation of the topography. Moreover, the computational time should be reduced with the 1D-2D coupled model, not only because a large number of $2 \mathrm{D}$ cells are eliminated from the computation but also because these cells are usually the responsible for the reduction of the time step size.

When focusing on the computational time, many simplified 1D-quasi 2D models were initially developed and applied to academic scenarios (Bladé et al., 1994), and realistic problems such as the Mekong river (Cunge, 1975) and the Severn River (Kuiry et al., 2010). All of them are based on the storage cell algorithm and the linking between the models is done by ensuring mass conservation. Instead of this, other authors proposed to connect both 1D and 2D hydrodynamic models by means of a weir equation (Lin et al., 2006; Villanueva and Wright, 2006), in which the water volume of both the $1 \mathrm{D}$ and the $2 \mathrm{D}$ domains were computed according to the level difference. Therefore, if the level in the main channel, predicted by the 1D model, exceeds that of the corresponding river embankment, the $1 \mathrm{D}$ and the $2 \mathrm{D}$ models will be appropriately linked by the mentioned weir equation.

In recent years, a few 1D-2D coupled models have been developed and applied to different configurations putting special emphasis on the link between the existing models. In (Gejadze and Monnier, 2007; Marin and Monnier, 2009) the idea of a 2D local zoom model superimposed over a $1 \mathrm{D}$ model was presented for simple problems, which was subsequently extended for complex geometries with discontinuous topographies between the models (FernándezNieto et al., 2010). However, these models had several disadvantages from the efficiency point of view such as the resolution required for the $2 \mathrm{D}$ model inside the river domain as well as the iterative algorithm needed to resolve the exchanges between the 1D/2D models. On the other hand, Finaud-Guyot et al. (2011) proposed a constant water surface elevation between the 1D cell and the $2 \mathrm{D}$ coupled cells, assuming that the exchange between the models is given by longitudinal momentum transfer that incorporates the presence of swirls between the models. However, the imposition of the same transversal velocity along the coupling zone seems to be no longer valid for complex flows.

Another 1D-2D coupled model was proposed by Morales-Hernández et al. (2013). It is based on common water surface elevation and/or average velocities in $x$ an $y$ direction. Two strategies that consist of conserving mass or conserving mass and momentum were considered and a procedure was provided to 
choose the correct strategy depending on the flow regime at the coupling zone and the number of required boundary conditions for each model. Although it is demonstrated to handle all kind of flow situations even in complex rivers, the imposition of the same flow variables at the coupling zone is improvable from the modelization point of view since it may distort the phenomena that occur at the 1D-2D frontier.

In this work, we propose the formulation of the coupling strategy without considering boundary conditions or exchange terms based on empirical laws: the interaction between the models will depend on the local Riemann Problem that arises across the edges that separate the $1 \mathrm{D}$ and the $2 \mathrm{D}$ models. A recent work developed by Goutal et al. (2014) links the models using a similar technique. Although they studied carefully the positivity preserving and the well-balanced property of the proposed scheme, the main drawback is that the transverse discharge for the $1 \mathrm{D}$ model is estimated via an iterative procedure. Moreover they proposed the use of an implicit 2D scheme for realistic applications, which may deteriorate the numerical results. In our work, a previous geometric coupling is mandatory to avoid the overlapping between the models. Therefore, as proposed in (Morales-Hernández et al., 2013), we define a coupling zone composed by one $1 \mathrm{D}$ cell and an exact number of $2 \mathrm{D}$ cells. Once the models are geometrically linked, it is necessary to define the fluxes for the 1D-1D, 2D-2D and 1D-2D edges, which will be solved by means of the Roe's cell-centered explicit upwind finite volume solver. For the 1D-2D interfaces, the idea is transforming the $1 \mathrm{D}$ quantities into $2 \mathrm{D}$ quantities and to solve the corresponding Riemann Problem as if it was a 2D-2D edge. Therefore, it is somehow necessary to estimate the $2 \mathrm{D}$ velocity field from the $1 \mathrm{D}$ discharge in order to be able to define the averaged quantities. The 1D-2D edges may govern the time step size hence they are incorporated in its computation in order to fulfil the stability condition. Moreover, the philosophy of the proposed 1D-2D coupled hydrodynamic model will be also extended to the solute transport equation, which can be decoupled in a conservative way by means of the corresponding numerical flux.

The paper is organised as follows: the shallow water equations as well as the numerical schemes are presented in both $1 \mathrm{D}$ and $2 \mathrm{D}$ versions. The solute transport equation is also displayed, which will be solved using a separate conservative technique. Then, the proposed 1D-2D coupled model is formulated, focusing on the importance of the geometric coupling and on the compact expression of the resulting numerical scheme. The solute transport equation is also included in the coupling procedure. Finally, the 1D-2D model is tested through different synthetic and realistic configurations. The accuracy and the computational time are evaluated in reference to the fully $2 \mathrm{D}$ model. 


\section{Governing equations and numerical schemes}

$2.11 \mathrm{D}$ shallow water equations

The equations for the 1D model can be described by means of mass and momentum conservation:

$$
\begin{gathered}
\frac{\partial \mathbf{U}(x, t)}{\partial t}+\frac{d \mathbf{F}(x, \mathbf{U})}{d x}=\mathbf{H}(x, \mathbf{U}) \\
\mathbf{U}=\left(\begin{array}{c}
A \\
Q
\end{array}\right), \quad \mathbf{F}=\left(\begin{array}{c}
Q \\
\frac{Q^{2}}{A}+g I_{1}
\end{array}\right), \quad \mathbf{H}=\left(\begin{array}{c}
0 \\
g\left[I_{2}+A\left(S_{0}-S_{f}\right)\right]
\end{array}\right)
\end{gathered}
$$

where $g=9.8 \mathrm{~m} / \mathrm{s}^{2}$ is the acceleration due to the gravity, $Q$ is the discharge, $A$ is the wetted cross section area and $S_{0}$ is the bed slope

$$
S_{0}=-\frac{\partial z_{b}}{\partial x}
$$

written in terms of the bed level $z_{b}$. Therefore, the water depth is denoted by $h=z_{s}-z_{b}$ being $z_{s}$ the water surface level. $S_{f}$ represents the friction stress modelled by the empirical Manning-Gauckler law

$$
S_{f}=\frac{Q^{2} n^{2}}{A^{2} R^{4 / 3}}
$$

where $R$ is the hydraulic radius and $n$ is the Manning roughness coefficient. $I_{1}$ accounts for hydrostatic pressure forces

$$
I_{1}(x, t)=\int_{0}^{h(x, t)}(h-\eta) \sigma(x, \eta) d \eta
$$

in a section of width $\sigma(x, \eta)$ at a position $\eta$ from the bottom (see Figure 1 ).

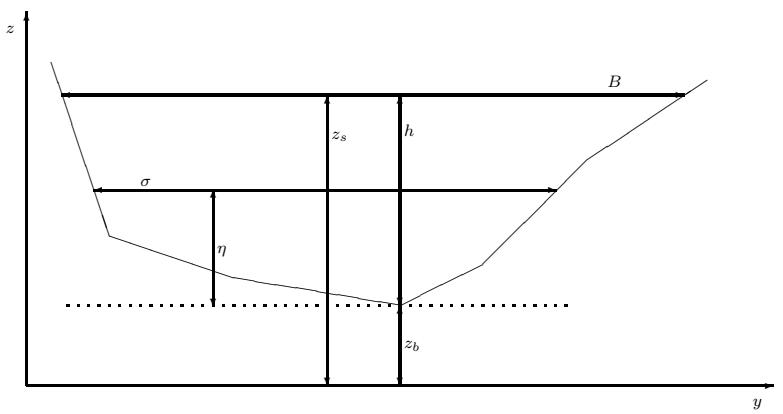

Fig. 1 Coordinate system in a cross section as used in the $1 \mathrm{D}$ model

With this definition, the cross sectional wetted area is expressed as follows: 


$$
A(x, t)=\int_{0}^{h(x, t)} \sigma(x, \eta) d \eta
$$

On the other hand, $I_{2}$ represents the pressure force due to the longitudinal width variations:

$$
I_{2}(x, t)=\int_{0}^{h(x, t)}(h-\eta) \frac{\partial \sigma(x, \eta)}{\partial x} d \eta
$$

The non-conservative system of equations is derived from (1) and (2), considering the following remark (Burguete and García-Navarro, 2001; MoralesHernández et al., 2013):

$$
\frac{d \mathbf{F}(x, \mathbf{U})}{d x}=\left.\frac{\partial \mathbf{F}(x, \mathbf{U})}{\partial x}\right|_{\mathbf{U}=\text { const }}+\left.\frac{\partial \mathbf{F}(x, \mathbf{U})}{\partial \mathbf{U}}\right|_{x=\text { const }} \frac{\partial \mathbf{U}(x, t)}{\partial x}
$$

and the so-called non-conservative source term $\mathbf{H}^{\prime}(x, \mathbf{U})$ appears naturally:

$$
\mathbf{H}^{\prime}(x, \mathbf{U})=\mathbf{H}(x, \mathbf{U})-\left.\frac{\partial \mathbf{F}(x, \mathbf{U})}{\partial x}\right|_{\mathbf{U}=\text { const }}
$$

\section{$2.22 \mathrm{D}$ shallow water equations}

The hyperbolic system of conservation laws that describes the $2 \mathrm{D}$ shallow water model is given by:

$$
\frac{\partial \mathbf{U}}{\partial t}+\frac{\partial \mathbf{F}(\mathbf{U})}{\partial x}+\frac{\partial \mathbf{G}(\mathbf{U})}{\partial y}=\mathbf{H}(\mathbf{U})
$$

where the conserved variables:

$$
\mathbf{U}=\left(h, q_{x}, q_{y}\right)^{T}
$$

are $h$ (the water depth) and $q_{x}=h u, q_{y}=h v$ (the unit discharges), being $(u, v)$ the averaged components of the velocity vector $\mathbf{u}$ along the $x$ and $y$ coordinates respectively. The fluxes of these conserved variables are:

$$
\mathbf{F}=\left(q_{x}, \frac{q_{x}^{2}}{h}+\frac{1}{2} g h^{2}, \frac{q_{x} q_{y}}{h}\right)^{T}, \quad \mathbf{G}=\left(q_{y}, \frac{q_{x} q_{y}}{h}, \frac{q_{y}^{2}}{h}+\frac{1}{2} g h^{2}\right)^{T}
$$

As in the 1D model, the source terms are related to the bed slope and friction stress

$$
\mathbf{H}=\left(0, g h\left(S_{0 x}-S_{f x}\right), g h\left(S_{0 y}-S_{f y}\right)\right)^{T}
$$

The bed slopes are expressed as the variation in the $x$ and $y$ direction of the bottom level $z$ 


$$
S_{0 x}=-\frac{\partial z}{\partial x}, \quad S_{0 y}=-\frac{\partial z}{\partial y}
$$

and the friction losses are written in terms of the Manning's roughness coefficient $n$ :

$$
S_{f x}=\frac{n^{2} u \sqrt{u^{2}+v^{2}}}{h^{4 / 3}}, \quad S_{f y}=\frac{n^{2} v \sqrt{u^{2}+v^{2}}}{h^{4 / 3}}
$$

\subsection{Numerical scheme}

It is feasible to express compactly the equations associated to the mentioned models and to apply an upwind first order explicit cell-centered finite volume scheme on both $1 \mathrm{D}$ and $2 \mathrm{D}$ shallow water equations:

$$
\frac{\partial \mathbf{U}}{\partial t}+\vec{\nabla} \mathbf{E}=\mathbf{S}
$$

where $\mathbf{E}=\mathbf{F}$ and $\mathbf{S}=\mathbf{H}^{\prime}$ for the $1 \mathrm{D}$ model and $\mathbf{E}=(\mathbf{F}, \mathbf{G})$ and $\mathbf{S}=\mathbf{H}$ in the $2 \mathrm{D}$ framework. The finite volume scheme is derived by integrating (16) in a computational cell $\Omega$ and by applying the Gauss' divergence theorem:

$$
\frac{\partial}{\partial t} \int_{\Omega} \mathbf{U} d \Omega+\int_{\Omega}(\vec{\nabla} \mathbf{E}) d \Omega=\int_{\Omega} \mathbf{S} d \Omega \Rightarrow \frac{\partial}{\partial t} \int_{\Omega} \mathbf{U} d \Omega+\oint_{T} \mathbf{E} \mathbf{n} d T=\int_{\Omega} \mathbf{S} d \Omega
$$

where $\mathbf{n}$ denotes the outward normal vector and $T=\partial \Omega$ is the surface surrounding the volume $\Omega$. The Jacobian $\mathbf{J}_{\mathbf{n}}$ of the normal flux $\mathbf{E} \mathbf{n}$ can be diagonalized

$$
\mathbf{J}_{\mathbf{n}}=\mathbf{P} \boldsymbol{\Lambda}_{\mathbf{n}} \mathbf{P}^{-1}, \quad \boldsymbol{\Lambda}_{\mathbf{n}}=\mathbf{P}^{-1} \mathbf{J}_{\mathbf{n}} \mathbf{P}
$$

where $\mathbf{P}$ is formed by the eigenvectors $\mathbf{e}^{m}$ of $\mathbf{J}_{\mathbf{n}}$ and the diagonal matrix $\boldsymbol{\Lambda}_{\mathbf{n}}$ is formed by the eigenvalues $\lambda^{m}$. Roe's linearization (Roe, 1981) is used to decouple the system (16) and to define locally an approximate matrix $\tilde{\mathbf{J}}_{\mathbf{n}, \mathbf{k}}$ at each computational wall $k$. The linearized eigenvalues $\tilde{\lambda}_{k}^{m}$ and eigenvectors $\tilde{\mathbf{e}}_{k}^{m}$ help us to express the differences in the conserved variables

$$
\delta \mathbf{U}_{k}=\mathbf{U}_{i}-\mathbf{U}_{j}=\sum_{m}(\tilde{\alpha} \tilde{\mathbf{e}})_{k}^{m}
$$

where $i$ and $j$ are the indexes of the cells that share the edge $k$. Following the same philosophy, the source terms can be projected onto the eigenvector basis and are also discretized at each wall

$$
\mathbf{S}_{k}=\sum_{m}(\tilde{\beta} \tilde{\mathbf{e}})_{k}^{m}
$$


It is worth clarifying that each model has a different set of eigenvectors $\tilde{\mathbf{e}}$ and they are carefully expressed in Appendix A for both numerical schemes. Moreover, it is well known that an upwind discretization of source terms provides an exact balance between fluxes and source terms that guarantees the C-property (Bermúdez and Vázquez-Cendón, 1994; Vázquez-Cendón, 1999) or steady-state equilibrium. Based on this fact, the 1D numerical scheme can be formulated for the updating of a cell $i$, as a sum of contributions from the left $(i-1 / 2)$ and right $(i+1 / 2)$ interfaces (Burguete and García-Navarro, 2001):

$$
\mathbf{U}_{i}^{n+1}=\mathbf{U}_{i}^{n}-\frac{\Delta t_{1 D}}{\Delta x}\left[\sum_{m}\left(\tilde{\lambda}^{+} \tilde{\gamma} \tilde{\mathbf{e}}\right)_{i-1 / 2}^{m}+\sum_{m}\left(\tilde{\lambda}^{-} \tilde{\gamma} \tilde{\mathbf{e}}\right)_{i+1 / 2}^{m}\right]^{n}
$$

where $m=2, \tilde{\gamma}_{i+1 / 2}^{m}=\left(\tilde{\alpha}-\frac{\tilde{\beta}}{\tilde{\lambda}}\right)_{i+1 / 2}^{m}$ contains in a compact expression the wave and source strengths and $\tilde{\lambda}_{i+1 / 2}^{ \pm m}=\frac{1}{2}(\tilde{\lambda} \pm|\tilde{\lambda}|)_{i+1 / 2}^{m}$ accounts for the upwind discretization. The Roe's linearized eigenvectors, eigenvalues and wave and source strengths are detailed in Appendix A. The explicit nature of the numerical scheme requires the time step $\Delta t$ to be restricted by the CFL condition:

$$
\Delta t_{1 D}=C F L \min _{m, k} \frac{\Delta x}{\left|\tilde{\lambda}_{k}^{m}\right|} \quad C F L \leq 1
$$

The expression for the 2D numerical upwind explicit scheme is completely analogous to that used for the 1D model: a cell $i$ with area $S_{i}$ is updated in time according to the in-going contributions (due to the fluxes and source terms) that arrive to the cell from the neighbouring walls

$$
\mathbf{U}_{i}^{n+1}=\mathbf{U}_{i}^{n}-\frac{\Delta t_{2 D}}{S_{i}} \sum_{k=1}^{N_{E}} \sum_{m}\left[\left(\tilde{\lambda}^{-} \tilde{\gamma} \tilde{\mathbf{e}}\right)_{k}^{m} l_{k}\right]^{n}
$$

where $m=3, N_{E}$ indicates the number of involved neighbouring walls $\left(N_{E}=3\right.$ for triangles), $l_{k}$ is the length of each computational wall and $\widetilde{\gamma}_{k}^{m}=\left(\widetilde{\alpha}-\frac{\widetilde{\beta}}{\widetilde{\lambda}}\right)_{k}^{m}$. The CFL condition for the time step size in $2 \mathrm{D}$ when considering unstructured meshes is:

$$
\Delta t_{2 D}=C F L \min _{m, k}\left\{\frac{\min \left(\chi_{i}, \chi_{j}\right)}{\left|\tilde{\lambda}_{k}^{m}\right|}\right\} \quad C F L \leq 1
$$

where $k$ loops over all the computational walls and $\chi_{i}$ is defined at each cell by using the area of the cell as well as the length of its $p$ neighbouring walls:

$$
\chi_{i}=\frac{S_{i}}{\max _{p=1, N_{E}} l_{p}}
$$


As in the 1D numerical scheme, the Roe's average values for the $2 \mathrm{D}$ numerical scheme are provided in Appendix A.

Realistic problems with strong bed variations and transient flow with wet/dry boundaries (specially in the 2D framework) can lead to small time step sizes and consequently to inefficient computations. After analysing the Augmented Riemann Problem, Murillo and García-Navarro (2010) proposed an efficient reduction of the source wave strength instead of reducing the time step size, preventing non-physical solutions and numerical instabilities and ensuring conservation, positivity of the water depth and the well-balanced property. In this work, these ideas are also adopted, guaranteeing these desirable features.

\subsection{Solute transport}

The interest of solute transport phenomena is usually put on the time evolution of a substance concentration within a complex hydrodynamic system, that is, given the solution concentration at a specific time and space, the aim is to know the spatial distribution of the solute concentrations at some future time. This physical process is modelled by means of the advection equation and can incorporate the effect of reaction (with the water and with other solutes) and solute diffusion. It can be coupled with both $1 \mathrm{D}$ and 2D hydrodynamic equations detailed above.

For the sake of clarity, the transport of only one solute is presented coupled to each model, even though it is possible to define more than one solute and to establish connections between them. It is worth noting that the solute transport equations will be expressed in a conservative form, assuming sudden variations on the fluid flow in space and time. In the present work, the solute transport does not include diffusion terms. However many strategies such as splitting and computing separately the advection and the diffusion terms or solving the diffusion implicitly have been reported to avoid small values in the time step size due to the combination of the CFL and Péclet number (Murillo et al., 2005, 2008).

The transport of a substance with a concentration of $\phi$ is expressed for the 1D framework:

$$
\frac{\partial(A \phi)}{\partial t}+\frac{\partial(Q \phi)}{\partial x}=-K A \phi
$$

while for the 2D model, it is written as follows:

$$
\frac{\partial(h \phi)}{\partial t}+\frac{\partial(h u \phi)}{\partial x}+\frac{\partial(h v \phi)}{\partial y}=-K h \phi
$$

In order to get a fully conservative method, the complete system including the hydrodynamic and the transport equations is considered. Mathematically, the complete system conserves the hyperbolicity property, implying the existence of a $3 \times 3$ or $4 \times 4$ Jacobian matrix for the $1 \mathrm{D}$ or the $2 \mathrm{D}$ model respectively. On this basis we can apply the procedure described above, allowing a Roe's 
local linearization and expressing the contributions that arrive to the cell as a sum of waves. This scheme guarantees the conservation but it can lead to unbounded values in the final solute concentration in extreme cases. A strategy that avoids these situations by enforcing a conservative redistribution of the solute mass fluxes was proposed in (Murillo et al., 2008).

However, it is feasible to decouple or separate the solute transport equation from the hydrodynamic system in a conservative way as in (Bristeau and Perthame, 2001), using an upwind mass flux $q^{\downarrow}$, directly related to the Roe's linearization. When decoupling the solute transport equation from the hydrodynamic system in that conservative way, the min-max property and the boundedness of the solution is guaranteed in the absence of source terms since the exact numerical flux is used. However, when reaction or diffusive terms are present, some corrections are needed in order to avoid spurious oscillations or unbounded solutions. As reported by Casulli et Zanolli (2005) using a high resolution scheme, the time step has to be restricted to achieve the min-max property. In this work, the ideas developed by Murillo and García-Navarro (2012) are applied. After considering all the set of possible approximate solutions in all kind of different flow situations for the volumetric concentration of the Augmented Riemann Problem, an upwind discretization and a correct strength estimation of the reaction terms is proposed and adopted in this work in order to ensure correct and bounded concentrations.

Therefore, in the 1D framework, the solute transport numerical scheme is formulated in a separate manner:

$$
(A \phi)_{i}^{n+1}=(A \phi)_{i}^{n}-\frac{\Delta t}{\Delta x}\left[(q \phi)_{i+1 / 2}^{\downarrow}-(q \phi)_{i-1 / 2}^{\downarrow}\right]
$$

where

$$
q_{i+1 / 2}^{\downarrow}=Q_{i}+\sum_{m=1}^{2}\left(\widetilde{\lambda}^{-} \widetilde{\gamma} \widetilde{\mathbf{e}}_{1}\right)_{i+1 / 2}^{m} \quad \phi_{i+1 / 2}^{\downarrow}=\left\{\begin{array}{cc}
\phi_{i} \text { if } q_{i+1 / 2}^{\downarrow}>0 \\
\phi_{i+1} \text { if } q_{i+1 / 2}^{\downarrow}<0
\end{array}\right.
$$

The 2D numerical scheme for the solute transport is analogously separated from the hydrodynamic equations using a conservative formulation:

$$
(h \phi)_{i}^{n+1}=(h \phi)_{i}^{n}-\frac{\Delta t}{A_{i}} \sum_{k=1}^{N_{E}}(q \phi)_{k}^{\downarrow} l_{k}
$$

where

$$
q_{k}^{\downarrow}=q_{i}+\sum_{m=1}^{3}\left(\widetilde{\lambda}^{-} \widetilde{\gamma} \widetilde{\mathbf{e}}_{1}\right)_{k}^{m} \quad \phi_{k}^{\downarrow}=\left\{\begin{array}{lll}
\phi_{i} & \text { if } q_{k}^{\downarrow}>0 \\
\phi_{j} & \text { if } & q_{k}^{\downarrow}<0
\end{array}\right.
$$




\section{RCE 1D-2D coupled model}

\subsection{Geometric linking and coupling strategy}

A new element of discretization for the 1D-2D coupled model is defined and called coupling zone. It will always contain one $1 \mathrm{D}$ cell and a number $\mathrm{NC}$ of $2 \mathrm{D}$ cells. Figure 2 shows two examples of coupling zones composed by only one 1D cell and some unstructured triangular 2D cells. As can be seen, two possible configurations may be defined with respect to the $1 \mathrm{D}$ model: frontal and lateral coupling. Moreover, the lateral configuration will contain left and right coupled cells.
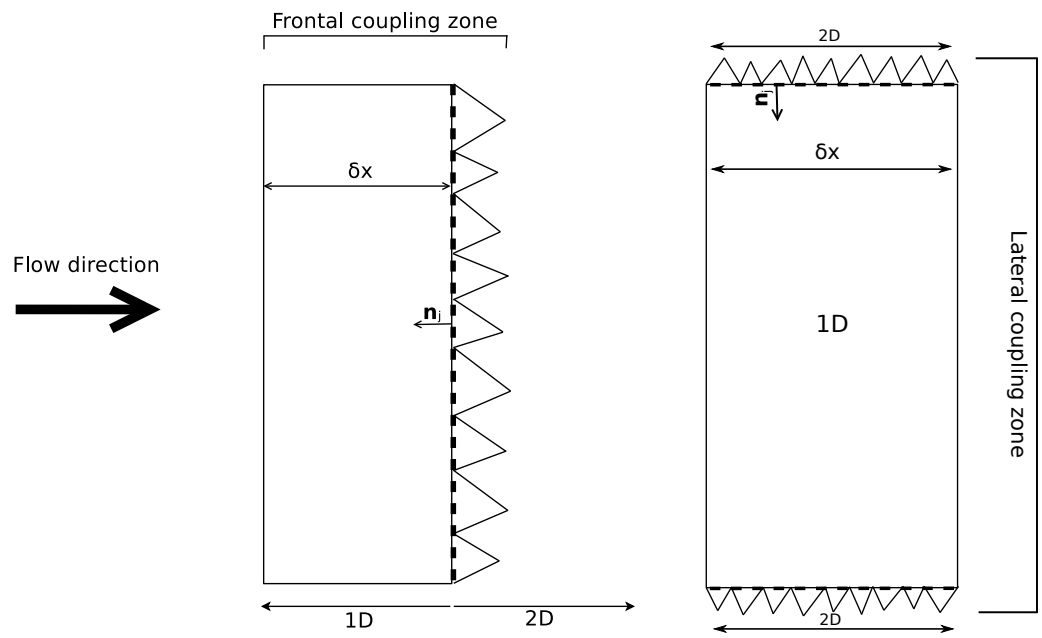

Fig. 2 Frontal and lateral coupling zones

The most direct implication of having an exact number of $2 \mathrm{D}$ cells at each coupling zone is the mesh discretization for the $2 \mathrm{D}$ model. The proposed strategy to achieve an adequate mesh for the 2D model is schematized in Figure 3 for the lateral configuration: once the full domain is characterized as a part of the $1 \mathrm{D}$ or of the $2 \mathrm{D}$ model (a), the $1 \mathrm{D}$ domain is discretized into a number of cells (b). This fact will provide a left bank and a right bank polylines (c) that will be used as input information for the $2 \mathrm{D}$ model. As each node defining the polylines belongs to the $2 \mathrm{D}$ model, an exact number of triangles will be defined for each 1D cell (d). Finally, the 1D cell and the 2D cells that belong to the same coupling zone are labelled with the same marker in order to simplify the linkage (e).

The identification of each coupling zone is done in the pre-process. Afterwards, the set of initial conditions for each model is applied and the computation starts. This approach is based on computing the fluxes at each interface, whether $1 \mathrm{D}-1 \mathrm{D}$ edge, $2 \mathrm{D}-2 \mathrm{D}$ edge or $1 \mathrm{D}-2 \mathrm{D}$ edge. As the computation of the 
(a)

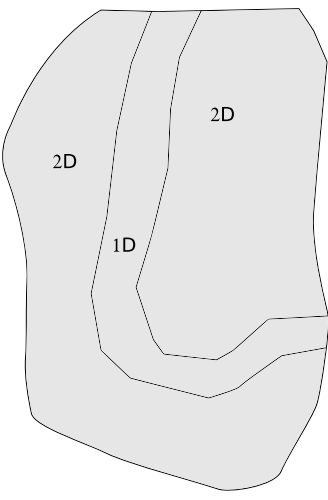

(b)

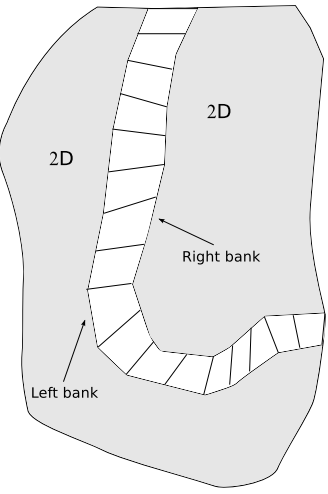

(c)

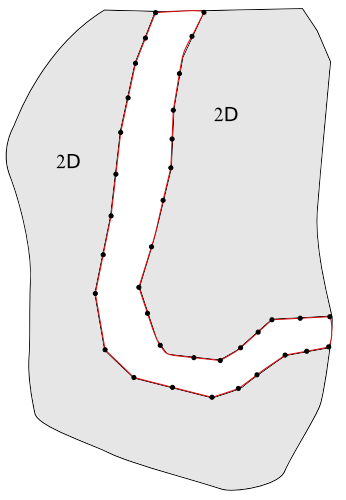

(d)

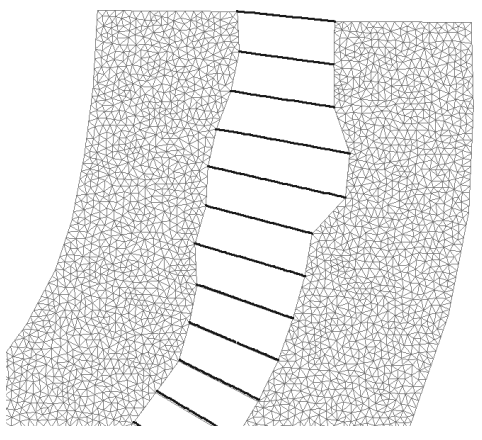

(e)

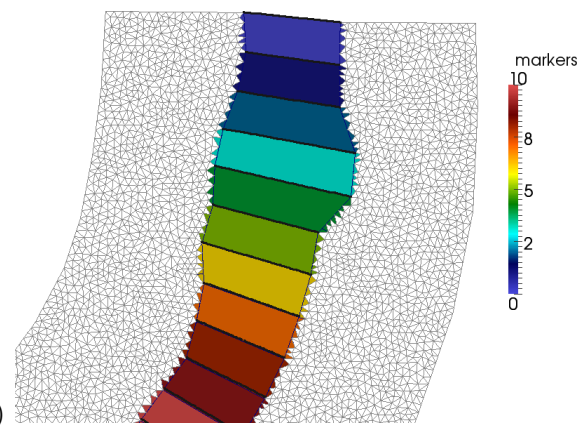

Fig. 3 Sketch of an adequate meshing procedure for the $2 \mathrm{D}$ domain in the coupled model

numerical fluxes for the 1D-1D and the 2D-2D edges is performed by using the conventional first order upwind scheme explained in (21) and (23) respectively, the focus will be put on the 1D-2D edges.

The strategy is based on the resolution of the local Riemann Problem at each $1 \mathrm{D}-2 \mathrm{D}$ interfaces, which requires the definition of discrete averaged values at each coupling edge. The idea developed in this work consists of transforming the 1D quantities into 2D quantities to solve the corresponding Riemann Problem as if it was a 2D-2D interface. Although the problem is undetermined in a certain way, estimations of the velocity field, the water depth and the bottom level are required to define the local Riemann Problem and to be able to define the edge averaged quantities. First, the bed level is set equal to the lowest point in the $1 \mathrm{D}$ cell and the water depth is chosen as the water depth of the 1D cell. Furthermore, the discharge in the 1D model, $Q_{1 D}$, can be converted into a vector according to the coupling zone angle $\theta$

$$
Q \rightsquigarrow\left(Q_{x}, Q_{y}\right)=(Q \cos \theta, Q \sin \theta)
$$

and consequently, the velocity vector $\mathbf{u}_{1 D}$ and the unit discharge vector $\mathbf{q}_{1 D}$ are easily estimated as: 


$$
\begin{gathered}
\mathbf{u}_{1 D}=\left(u_{1 D}, v_{1 D}\right)=\left(\left(\frac{Q}{A}\right)_{1 D} \cos \theta,\left(\frac{Q}{A}\right)_{1 D} \sin \theta\right) \\
\mathbf{q}_{1 D}=\left(q_{x 1 D}, q_{y_{1 D}}\right)=\left(\left(\frac{Q}{B}\right)_{1 D} \cos \theta,\left(\frac{Q}{B}\right)_{1 D} \sin \theta\right)
\end{gathered}
$$

where $A_{1 D}$ and $B_{1 D}$ are the wetted area and the top width respectively. It is worth noting that, in the case of the lateral configuration, this angle is characterized for each coupling zone side $\theta_{L}$ or $\theta_{R}$ (left or right). These angles (Figure 4) can be geometrically computed from the $x, y$ coordinates defining each 1D cell $\left(\mathbf{p}_{L}, \mathbf{r}_{L}, \mathbf{p}_{R}, \mathbf{r}_{R}\right)$ :

$$
\begin{gathered}
\theta_{L}=\arccos \frac{r_{L x}-p_{L x}}{\sqrt{\left(r_{L x}-p_{L x}\right)^{2}+\left(r_{L y}-p_{L y}\right)^{2}}} \\
\theta_{R}=\arccos \frac{r_{R x}-p_{R x}}{\sqrt{\left(r_{R x}-p_{R x}\right)^{2}+\left(r_{R y}-p_{R y}\right)^{2}}}
\end{gathered}
$$

Therefore, each Riemann Problem can be projected over the direction $x^{\prime}$ given by the normal vector $\mathbf{n}_{\kappa}$ at each $1 \mathrm{D}-2 \mathrm{D}$ wall or edge $\kappa$ (see Figure 4 )

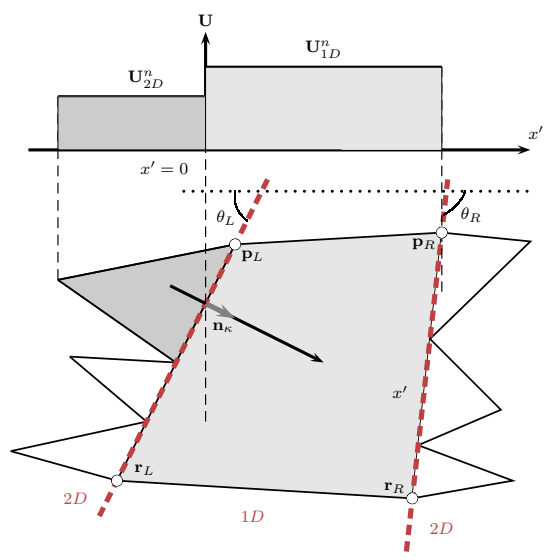

Fig. 4 Riemann problem in the 1D-2D edge

The average quantities that allow to define the local Riemann Problem are consequently written as follows:

$\tilde{u}=\frac{\sqrt{h_{2 D}} u_{2 D}+\sqrt{h_{1 D}} u_{1 D}}{\sqrt{h_{2 D}}+\sqrt{h_{1 D}}}, \quad \tilde{v}=\frac{\sqrt{h_{2 D}} v_{2 D}+\sqrt{h_{1 D}} v_{1 D}}{\sqrt{h_{2 D}}+\sqrt{h_{1 D}}}, \quad \tilde{c}=\sqrt{g \frac{h_{2 D}+h_{1 D}}{2}}$ 
where $\delta h=h_{1 D}-h_{2 D}, \delta z=z_{1 D}-z_{2 D}, \delta q_{x}=q_{x_{1 D}}-q_{x_{2 D}}, \delta q_{y}=q_{y_{1 D}}-$ $q_{y_{2} D}, \tilde{\mathbf{u}} \cdot \mathbf{n}=\tilde{u} n_{x}+\tilde{v} n_{y}, \delta \mathbf{q} \cdot \mathbf{n}=\delta q_{x} n_{x}+\delta q_{y} n_{y}$ and the average states at each coupled $1 \mathrm{D}-2 \mathrm{D}$ wall $\kappa$ are represented with the tilde variables. The local eigenvalues $\widetilde{\lambda}^{m}$ and eigenvectors $\widetilde{\mathbf{e}}^{m}$, as well as the contributions due to the fluxes and the source terms are defined as follows:

$$
\begin{gathered}
\mathbf{e}_{1}=\left(\begin{array}{c}
1 \\
\tilde{u}-\tilde{c} n_{x} \\
\tilde{v}-\tilde{c} n_{y}
\end{array}\right), \quad \mathbf{e}_{2}=\left(\begin{array}{c}
0 \\
-\tilde{c} n_{y} \\
\tilde{c} n_{x}
\end{array}\right), \quad \mathbf{e}_{3}=\left(\begin{array}{c}
1 \\
\tilde{u}+\tilde{c} n_{x} \\
\tilde{v}+\tilde{c} n_{y}
\end{array}\right), \\
\lambda_{1}=\tilde{\mathbf{u}} \cdot \mathbf{n}-\tilde{c}, \quad \lambda_{2}=\tilde{\mathbf{u}} \cdot \mathbf{n}, \quad \lambda_{3}=\tilde{\mathbf{u}} \cdot \mathbf{n}+\tilde{c} \\
\left.\left.\tilde{\alpha}_{1}=\frac{\delta h}{2}-\frac{1}{2 \tilde{c}}(\delta \mathbf{q} \cdot \mathbf{n}-\tilde{\mathbf{u}} \cdot \mathbf{n} \delta h), \quad \tilde{\alpha}_{2}=\frac{1}{\tilde{c}}\left[\delta q_{y}-\tilde{v} \delta h\right) n_{x}-\left(\delta q_{x}-\tilde{u} \delta h\right) n_{y}\right)\right], \\
\tilde{\alpha}_{3}=\frac{\delta h}{2}+\frac{1}{2 \tilde{c}}(\delta \mathbf{q} \cdot \mathbf{n}-\tilde{\mathbf{u}} \cdot \mathbf{n} \delta h), \\
\tilde{\beta}_{1}=\frac{g \tilde{h}}{2 c}\left(\delta z+S_{f, \mathbf{n}}\right), \quad \tilde{\beta}_{2}=0, \quad \tilde{\beta}_{3}=-\tilde{\beta}_{1}
\end{gathered}
$$

Note that the friction term requires the definition of an average Manning roughness coefficient between cells $1 \mathrm{D}$ and $2 \mathrm{D}$ and also the computation of the normal distance between the centroid of the involved cells.

Once the averaged quantities for the 1D-2D edges are defined, the time step size is a question of interest. It should be chosen as the minimum of the time step sizes of the three interfaces:

$$
\Delta t=\min \left(\Delta t_{1 D}, \Delta t_{2 D}, \Delta t_{1 D 2 D}\right)
$$

where $\Delta t_{1 D}$ and $\Delta t_{2 D}$ are the time step sizes for the 1D-1D edges and for the 2D-2D edges, as defined in (22) and (24) respectively. Given a 1D-2D edge $\kappa$ with length $l_{\kappa}$, separating a $1 \mathrm{D}$ cell and a $2 \mathrm{D}$ adjacent cell with area $S_{2 D}$, $\Delta t_{1 D 2 D}$ is defined as:

$$
\Delta t_{1 D 2 D}=\mathrm{CFL} \min _{m, \kappa} \frac{\chi_{\kappa}}{\left|\tilde{\lambda}_{\kappa}^{m}\right|} \quad \chi_{\kappa}=\min \left(\Delta x_{1 D}, S_{2 D} / l_{\kappa}\right)
$$

with $\kappa$ runs over the coupling edges. Once the contributions due to the fluxes and source terms are sent, the conserved variables are updated and the time is increased as usual. The procedure can be summarized in Figure 5, where a flowchart of the RCE 1D-2D model is included.

\subsection{Extended numerical scheme}

The numerical resolution of the RCE coupled model is indeed composed by three sub-solvers, each one in charge of a different type of edges. As mentioned 


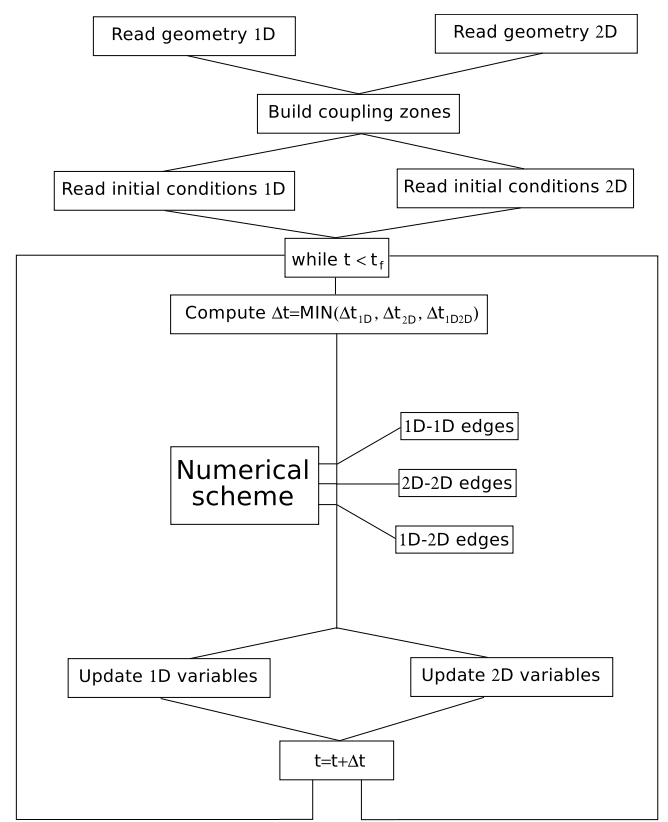

Fig. 5 Flowchart of the RCE 1D-2D scheme

above, the 1D-1D edges as well as the 2D-2D edges are solved as usual, i.e., sending the information as if there were not any connection between the models. The third one involves the 1D-2D interfaces and is fairly similar to the resolution of the 2D-2D edges. Figure 6 shows a sketch of the contributions from each type of edge in the RCE 1D-2D coupled model.

The expression of the numerical scheme for the updating of a $2 \mathrm{D}$ triangular cell, including the contributions from the 2D-2D edges $k$ as well as those coming from the $1 \mathrm{D}-2 \mathrm{D}$ edges $\kappa$ is

$$
\mathbf{U}_{i}^{n+1}=\mathbf{U}_{i}^{n}-\underbrace{\frac{\Delta t}{S_{i}} \sum_{k=1}^{N_{E}} \sum_{m}\left[\left(\tilde{\lambda}^{-} \tilde{\gamma} \tilde{\mathbf{e}}\right)_{k}^{m} l_{k}\right]^{n}}_{2 D-2 D \text { edges }}-\underbrace{\frac{\Delta t}{S_{i}} \sum_{m}\left[\left(\tilde{\lambda}^{-} \tilde{\gamma} \tilde{\mathbf{e}}\right)_{\kappa}^{m} l_{\kappa}\right]^{n}}_{1 D-2 D \text { edges }}
$$

where $m=1 . .3, \widetilde{\gamma}_{\kappa}^{m}=\left(\widetilde{\alpha}-\frac{\widetilde{\beta}}{\widetilde{\lambda}}\right)_{\kappa}^{m}$ and $N_{E}$ represents the number of neighbouring $2 \mathrm{D}$ cells. In the case of inner cells, $N_{E}=3$; otherwise $N_{E}=2$ if the cell is a non-corner boundary cell and $N_{E}=1$ if it is a corner boundary cell.

On the contrary, the $1 \mathrm{D}$ numerical scheme including both the contributions of the 1D-1D and the 1D-2D edges must be split into two expressions, representing the updating of the wetted area: 


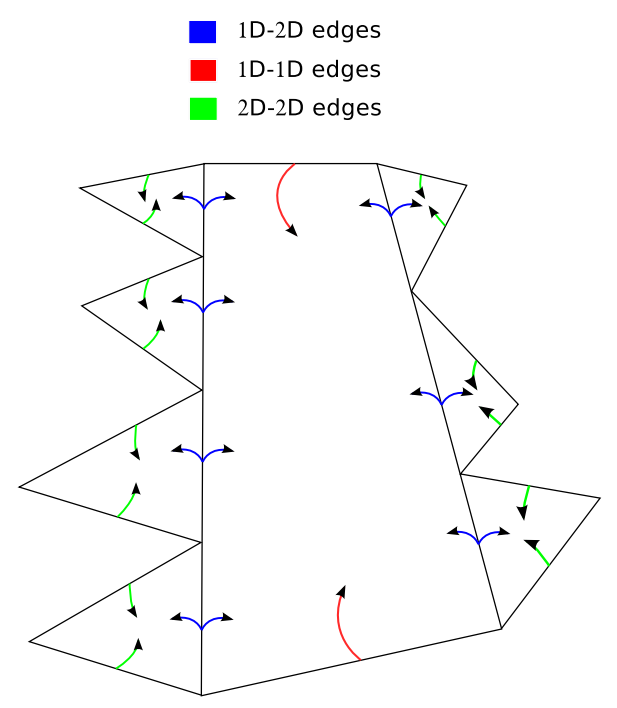

Fig. 6 Sketch of the contributions in the RCE 1D-2D model

$$
\begin{aligned}
A_{i}^{n+1}=A_{i}^{n} & -\underbrace{\frac{\Delta t}{\Delta x}\left[\sum_{m_{1}}\left(\tilde{\lambda}^{+} \tilde{\gamma} \tilde{e}_{1}\right)_{i-1 / 2}^{m_{1}}+\sum_{m_{1}}\left(\tilde{\lambda}^{-} \tilde{\gamma} \tilde{e}_{1}\right)_{i+1 / 2}^{m_{1}}\right]^{n}}_{1 D-1 D \text { edges }} \\
& -\underbrace{\frac{\Delta t}{\Delta x} \sum_{i=0}^{N_{C}} \sum_{m_{2}=1}^{3}\left[\left(\tilde{\lambda}^{-} \tilde{\gamma} \tilde{e}_{1}\right)_{\kappa}^{m_{2}} l_{\kappa}\right]^{n}}_{1 D-2 D \text { edges }}
\end{aligned}
$$

and of the discharge:

$$
\begin{gathered}
Q_{i}^{n+1}=Q_{i}^{n}-\underbrace{\frac{\Delta t}{\Delta x}\left[\sum_{m_{1}}\left(\tilde{\lambda}^{+} \tilde{\gamma} \tilde{e}_{2}\right)_{i-1 / 2}^{m_{1}}+\sum_{m_{1}}\left(\tilde{\lambda}^{-} \tilde{\gamma} \tilde{e}_{2}\right)_{i+1 / 2}^{m_{1}}\right]^{n}}_{1 D-1 D \text { edges }} \\
-\underbrace{\frac{\Delta t}{\Delta x} \sum_{i=0}^{N_{C}} \sum_{m_{2}=1}^{3}\left[\left(\tilde{\lambda}^{-} \tilde{\gamma} \tilde{e}_{2}\right)_{\kappa}^{m_{2}} l_{\kappa}\right]^{n} \cos \theta-\frac{\Delta t}{\Delta x} \sum_{i=0}^{N_{C}} \sum_{m_{2}=1}^{3}\left[\left(\tilde{\lambda}^{-} \tilde{\gamma}^{\tilde{e}_{3}}\right)_{\kappa}^{m_{2}} l_{\kappa}\right]^{n} \sin \theta}_{1 D-2 D \text { edges }}
\end{gathered}
$$

where $m_{1}=1 . .2$ and $N_{C}$ is the number of adjacent $2 \mathrm{D}$ cells associated to the $1 \mathrm{D}$ cell in the coupling zone. It should be noted that, in the lateral configuration, the contribution from the $1 \mathrm{D}-2 \mathrm{D}$ edges are non-zero when an overflow 
occurs. Otherwise, solid wall or zero flow has to be imposed over the 2D cells that could have water depth greater than zero.

The well-balanced property (still water) for the 1D-2D coupled model is guaranteed and can be easily deduced from a simple computation. Let consider the following initial data:

$$
(h+z)_{1 D}=(h+z)_{2 D} \quad u_{1 D}=u_{2 D}=0
$$

At the $1 \mathrm{D}-2 \mathrm{D}$ edge $\kappa$, the total contributions are:

$$
\begin{aligned}
& \lambda^{1} \alpha^{1}-\beta^{1}=-\tilde{c} \frac{\delta h}{2}-\frac{g \tilde{h}}{2 \tilde{c}} \delta z=\frac{-\tilde{c}^{2} \delta h+g \tilde{h} \delta h}{2 \tilde{c}}=0 \\
& \lambda^{2} \alpha^{2}-\beta^{2}=0 \\
& \lambda^{3} \alpha^{3}-\beta^{3}=\tilde{c} \frac{\delta h}{2}+\frac{g \tilde{h}}{2 \tilde{c}} \delta z=\frac{\tilde{c}^{2} \delta h-g \tilde{h} \delta h}{2 \tilde{c}}=0
\end{aligned}
$$

hence the still-water property is satisfied. On the other hand, the ideas developed in (Murillo and García-Navarro, 2010) for the positivity preserving property of the water depth are also applied in this work for the 1D-2D coupled model. As a result, it is feasible to enforce the non-negative value of the intermediate states arising from the Riemann Problem for the 1D-2D edges by limiting the amount of source in the case of extreme slopes or friction terms. With this consideration, a positive value of the water depth is always guaranteed.

\subsection{Solute transport}

The RCE strategy to couple the $1 \mathrm{D}$ and the $2 \mathrm{D}$ models can be conveniently extended to the solute transport equations using the conservative approach explained in 2.4. Let consider a solute with a concentration $\phi$. The numerical flux for the solute equation can be written for the 1D-2D edges $\kappa$

$$
q_{\kappa}^{\downarrow}=q_{2 D}+\sum_{m=1}^{3}\left(\widetilde{\lambda}^{-} \widetilde{\gamma} \widetilde{\mathbf{e}}_{1}\right)_{\kappa}^{m} \quad \phi_{\kappa}^{\downarrow}= \begin{cases}\phi_{2 D} & \text { if } q_{\kappa}^{\downarrow}>0 \\ \phi_{1 D} & \text { if } q_{\kappa}^{\downarrow}<0\end{cases}
$$

where $q_{2 D}=\left(q_{x} n_{x}+q_{y} n_{y}\right)_{2 D}$ is the normal unit discharge of the $2 \mathrm{D}$ cell. The complete 2D numerical scheme, combining the contributions coming from the $2 \mathrm{D}-2 \mathrm{D}$ edges and those arriving from the $1 \mathrm{D}-2 \mathrm{D}$ edges, is formulated in a conservative way

$$
(h \phi)_{i}^{n+1}=(h \phi)_{i}^{n}-\underbrace{\frac{\Delta t}{A_{i}} \sum_{k=1}^{N_{E}}(q \phi)_{k}^{\downarrow} l_{k}}_{2 D-2 D \text { edges }}-\underbrace{\frac{\Delta t}{A_{i}}(q \phi)_{\kappa}^{\downarrow} l_{\kappa}}_{1 D-2 D \text { edges }}
$$


The 1D scheme can be expressed compactly again, adding to (28) the contributions from the $N_{C}$ adjacent 2D cells associated to the 1D cell through the corresponding coupling zone:

$$
(A \phi)_{i}^{n+1}=(A \phi)_{i}^{n}-\underbrace{\frac{\Delta t}{\Delta x}\left[(q \phi)_{i+1 / 2}^{\downarrow}-(q \phi)_{i-1 / 2}^{\downarrow}\right]}_{1 D-1 D \text { edges }}-\underbrace{\frac{\Delta t}{\Delta x} \sum_{\kappa=1}^{N_{C}}(q \phi)_{\kappa}^{\downarrow} l_{\kappa}}_{1 D-2 D \text { edges }}
$$

The maximum principle is again satisfied for the solute transport equation since the same techniques described in (Murillo and García-Navarro, 2012) are used for the 1D-2D coupled interfaces.

\section{Numerical results}

The main objective of this section is to test the proposed RCE 1D-2D coupled model by means of two test cases that include the presence of the solute transport equation. The numerical results achieved by the mentioned 1D-2D coupled model will be validated in comparison with a fully $2 \mathrm{D}$ model in terms of longitudinal profiles and local measurements at certain points.

\subsection{Test case 1: channel with a lateral floodplain area}

This test case corresponds to that presented in (Morales-Hernández et al., 2013), where a main trapezoidal channel is connected laterally with a floodplain area. The length of the channel is $2000 \mathrm{~m}$ separated in three regions: in the first and the third zones, the left and right banks are six meters high while in the second zone the left bank is eliminated to link the channel with the floodplain. Both the channel and the floodplain have a $x$ slope of 1/1000 (see Figure 7).

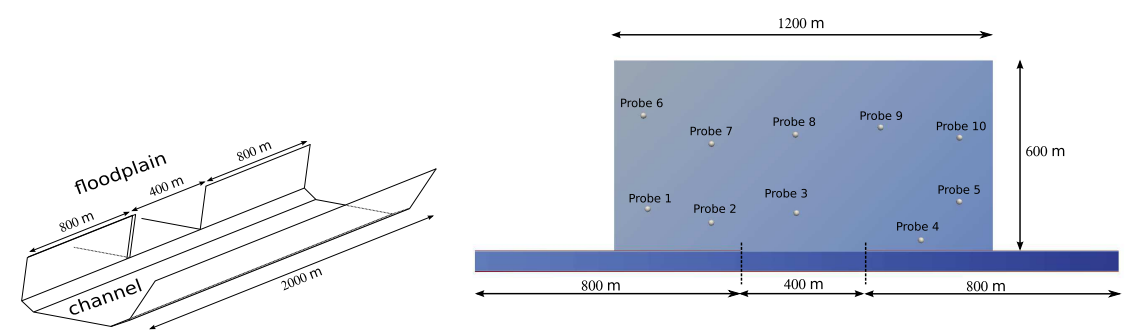

Fig. 7 Test case 1: description and location of the probes 


\subsubsection{Well-balanced property: lake at rest}

The first configuration is introduced in order to check the well-balanced property with null velocity. Therefore, an initial condition given by:

$$
h+z=3.5 \quad(u, v)_{2 D}=Q_{1 D}=0
$$

is enforced all over the domain. As previously deduced analytically, the initial configuration should be conserved after applying the mentioned RCE 1D-2D coupled. Figure 8 shows the results after $200 \mathrm{~s}$ for the water level and velocity magnitude (upper and lower respectively). On the left side of the figure only the wet domain is represented while on the right side the discretized black line is plotted for the corresponding variables.
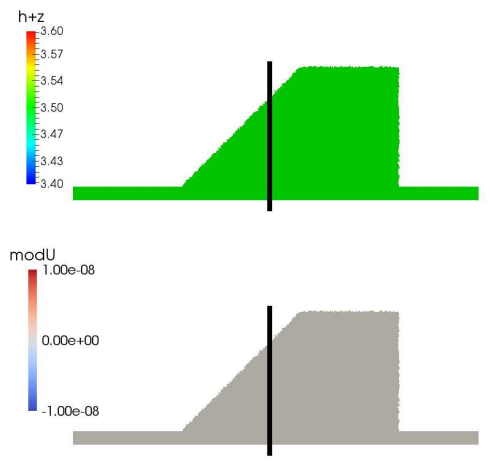

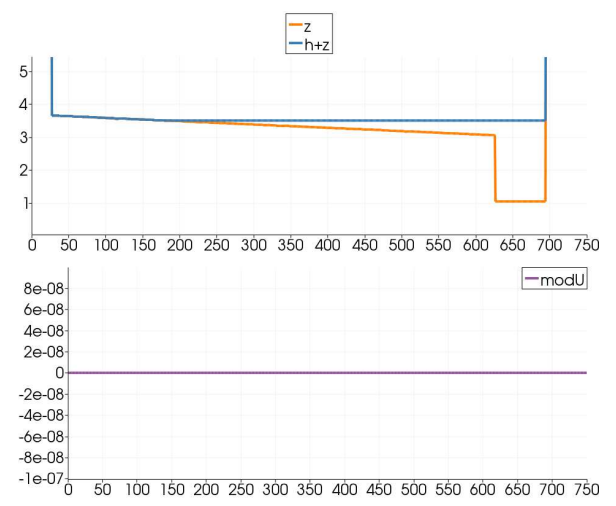

Fig. 8 Test case 1: Still water at $t=200$ s. Water surface level (upper) and velocity magnitude (lower)

As expected, the surface level as well as the null velocity are maintained and no spurious oscillations are introduced as a result of the coupling strategy.

\subsubsection{Convergence to the steady state}

From an initial condition of $h=2 \mathrm{~m}$ inside the main channel, a constant discharge of $600 \mathrm{~m}^{3} / \mathrm{s}$ is introduced as inlet boundary condition and both the $2 \mathrm{D}$ and the RCE $1 \mathrm{D}-2 \mathrm{D}$ models are run until the convergence to the steady state is reached. A gauging curve is imposed as outlet boundary condition. The numerical results in terms of longitudinal profile along the centerline of the channel as well as the evolution in time of different probes inside the floodplain (Figure 7, right) are examined for the RCE 1D-2D model in comparison with the 2D model. According to (Morales-Hernández et al., 2013) and regarding the interpretation in each model, the Manning's roughness coefficient in the main channel is chosen as $n=0.01605 \mathrm{~s} / \mathrm{m}^{1 / 3}$ for the $1 \mathrm{D}-2 \mathrm{D}$ model and $n=0.015 \mathrm{~s} / \mathrm{m}^{1 / 3}$ for the $2 \mathrm{D}$ model. In the floodplain, both models have the same coefficient $n=0.03 \mathrm{~s} / \mathrm{m}^{1 / 3}$. 
The longitudinal profile when the steady state is reached is plotted in Figure 9. As can be observed, the profile obtained by the RCE 1D-2D model behaves as well as that achieved by the fully 2D model although it is slightly overestimated in certain zones.

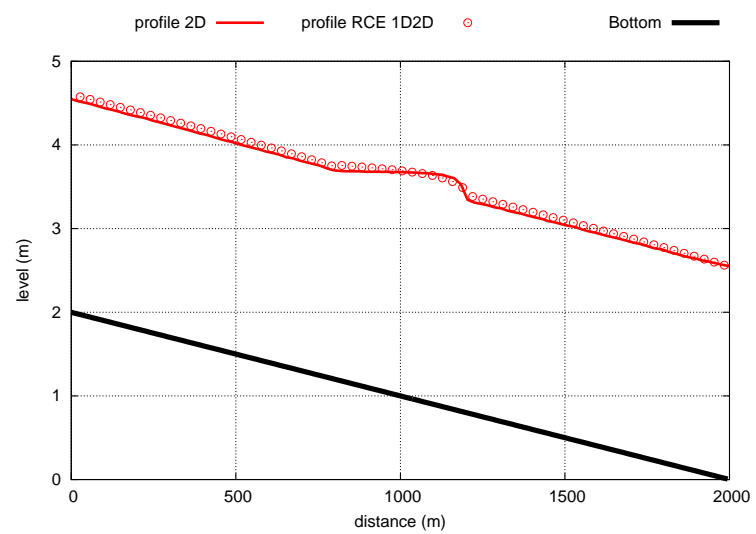

Fig. 9 Test case 1: longitudinal profile along the channel

On the other hand, the convergence to the steady state is analyzed by means of Figures 10 and 11 (probes 1-5 and 6-10, respectively), where the comparison in terms of time evolution of the water depth is carried out. The unsteady character of the flow when the floodplain is inundated is well captured by the RCE 1D-2D model and the levels achieved when the steady state is reached are almost the same for both models in all the gauging points. As an example, probe 6 is always dry during all the event.

\subsubsection{Solute transport}

The implementation of the solute transport equation in the RCE 1D-2D coupled model is also tested by means of the same trapezoidal channel, with a simplified Nitrogen Water Quality Model involving nitrate $\left(\mathrm{NO}_{3}\right)$ and ammonium $\left(\mathrm{NH}_{4}\right)$. These substances are interdependent and usually, the $\mathrm{NO}_{3}$ concentration increases in the detriment of $\mathrm{NH}_{4}$. In fact, the solute equations can be written for the $1 \mathrm{D}$ configuration:

$$
\begin{gathered}
\frac{\partial\left(A \phi_{1}\right)}{\partial t}+\frac{\partial\left(Q \phi_{1}\right)}{\partial x}=-K_{1} A \phi_{1} \\
\frac{\partial\left(A \phi_{2}\right)}{\partial t}+\frac{\partial\left(Q \phi_{2}\right)}{\partial x}=K_{1} A \phi_{1}-K_{2} A \phi_{2}
\end{gathered}
$$

and for the 2D framework: 


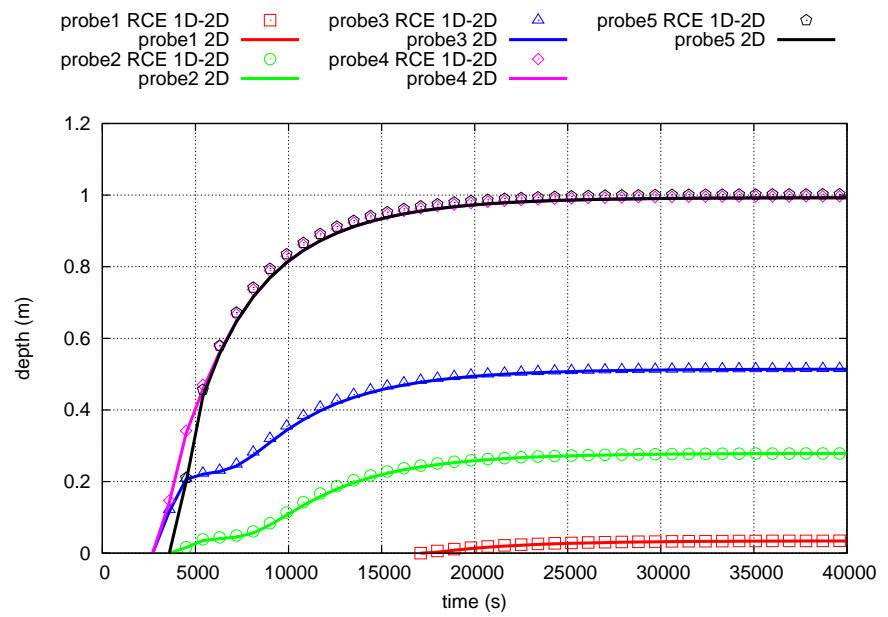

Fig. 10 Test case 1: time evolution of water depth at probes 1-5. 1D-2D (points), fully 2D (lines)

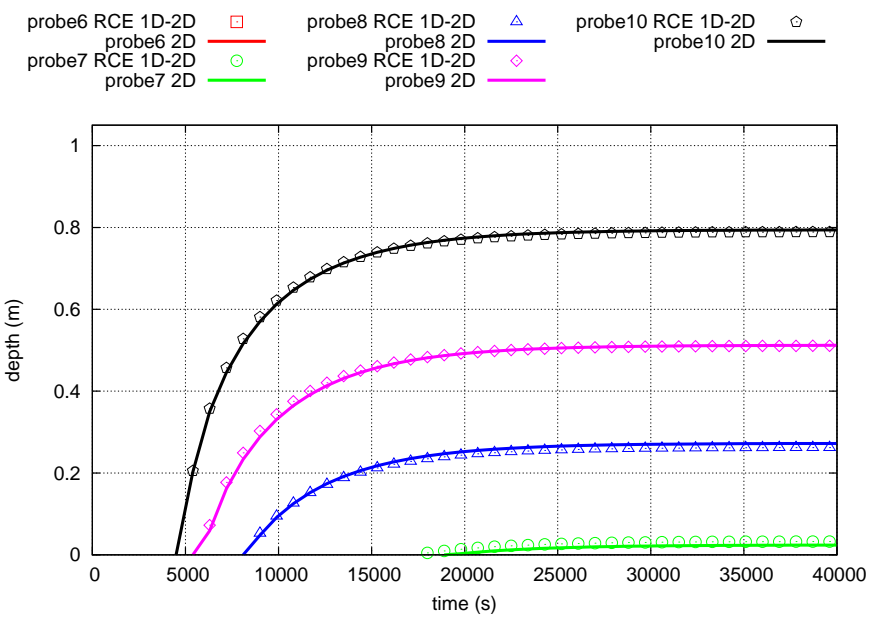

Fig. 11 Test case 1: time evolution of water depth at probes 6-10. 1D-2D (points), fully 2D (lines)

$$
\begin{gathered}
\frac{\partial\left(h \phi_{1}\right)}{\partial t}+\frac{\partial\left(h u \phi_{1}\right)}{\partial x}+\frac{\partial\left(h v \phi_{1}\right)}{\partial y}=-K_{1} A \phi_{1} \\
\frac{\partial\left(h \phi_{2}\right)}{\partial t}+\frac{\partial\left(h u \phi_{2}\right)}{\partial x}+\frac{\partial\left(h v \phi_{2}\right)}{\partial y}=K_{1} A \phi_{1}-K_{2} A \phi_{2}
\end{gathered}
$$

where $\phi_{1}, \phi_{2}$ are the ammonium and nitrate concentration respectively and $K_{1}, K_{2}$ are the uptake constants expressed as follows:

$$
K_{1}=K_{N_{4}} 10^{0.0293 T} \quad K_{2}=K_{N O_{3}} 1.069810^{0.0293 T}
$$


being $T=20{ }^{\circ} \mathrm{C}$ the water temperature $\left({ }^{\circ} \mathrm{C}\right)$ and $K_{\mathrm{NH}_{4}}, K_{\mathrm{NO}_{3}}$ the nitrification and denitrification rate coefficients.

Following the previous geometric configuration, a constant discharge of $600 \mathrm{~m}^{3} / \mathrm{s}$ with constant ammonium $\left(\phi_{1}=0.2\right)$ and nitrate concentration $\left(\phi_{2}=3.0\right)$ is introduced as inlet boundary condition. Once the steady state is reached, a rapid change in both concentrations $\left(\phi_{1}=2.0, \phi_{2}=5.5\right)$ is injected to the system emulating a dump upstream the channel. After one hour, the concentrations turn back to their initial configuration. Figure 12 shows the evolution in time of the inlet ammonium and nitrate concentration.

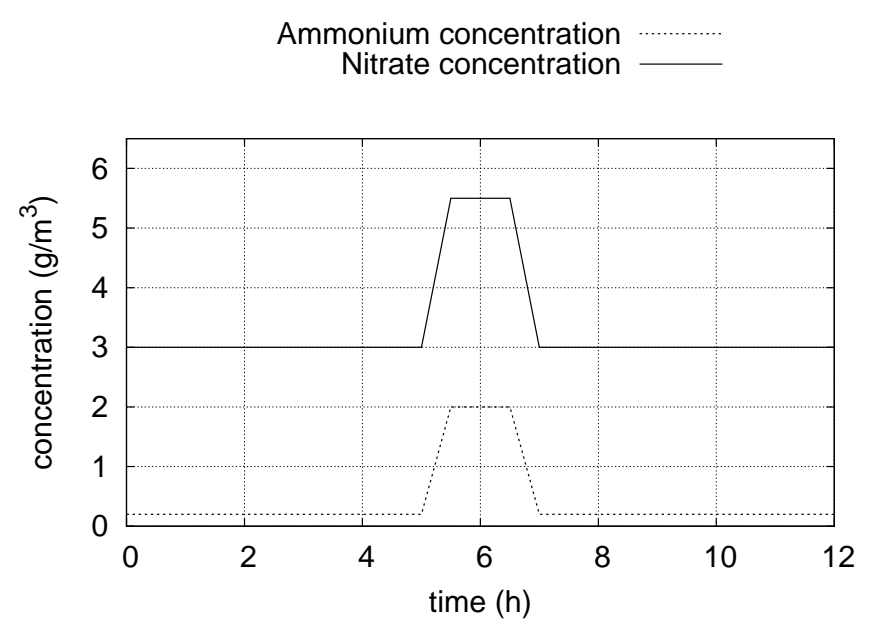

Fig. 12 Test case 1: Evolution in time of the inlet nitrate and ammonium concentration

The simulation time is $12 \mathrm{~h}$. Figure 13 shows two snapshots at $\mathrm{t}=7.5 \mathrm{~h}$ for the ammonium concentration (left) and at $\mathrm{t}=12 \mathrm{~h}$ for the nitrate concentration (right). The numerical schemes used are the fully 2D model (upper) and the RCE 1D-2D model (lower). As displayed in these figures, the RCE 1D-2D coupled model is qualitative able to approximate the results achieved by the 2D model.

In order to corroborate this conjecture, the evolution in time of each probe defined for the system in Figure 7 (right) is again registered for the ammonium and for the nitrate concentration in Figures 14 and 15 respectively.

As observed, the RCE 1D-2D model when considering the solute transport equation registers some delays at probes 8 and 9 and arrives earlier than the $2 \mathrm{D}$ model at probe 3 . These wrong estimations can be attributed to the velocity field for the RCE $1 \mathrm{D}-2 \mathrm{D}$ model, that is slightly different from the $2 \mathrm{D}$ complete model and does not capture the partial bidimensionality of the main channel an the end of the floodplain. The solute transport equation is indeed very sensitive to the velocity field hence little changes in these variable may produce large changes in the spatial and temporal distribution of the solute. 


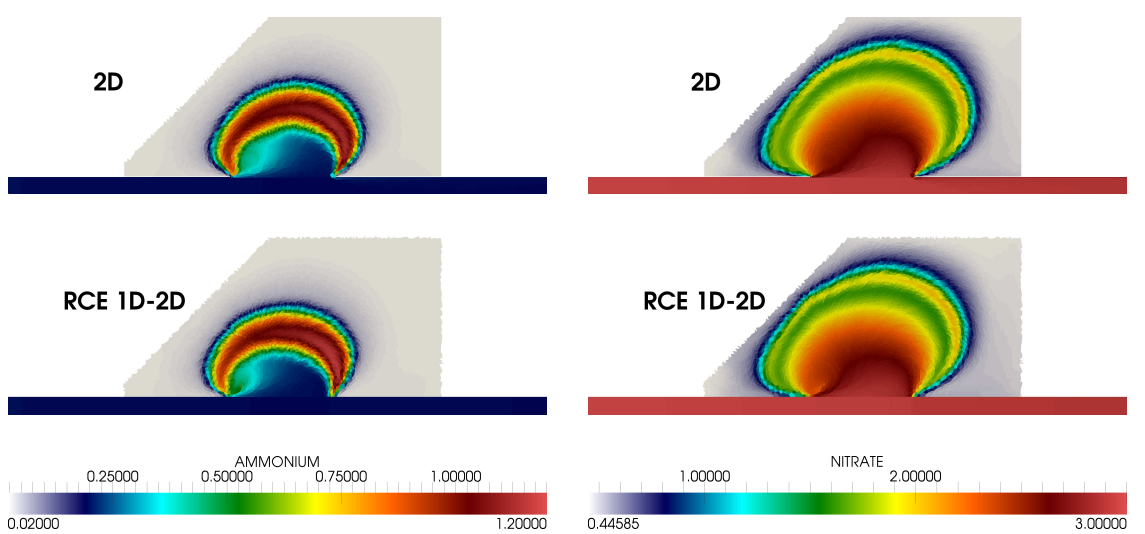

Fig. 13 Test case 1: Snapshots at $\mathrm{t}=7.5 \mathrm{~h}$ for the ammonium concentration (left) and at $\mathrm{t}=12 \mathrm{~h}$ for the nitrate concentration (right). Fully 2D model (upper) and RCE 1D-2D model (lower)
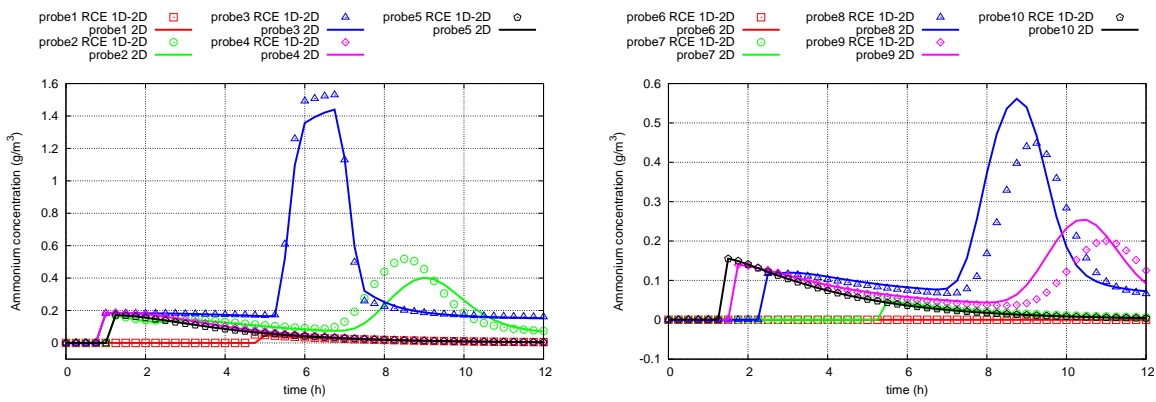

Fig. 14 Test case 1: Numerical solution of the time evolution of the ammonium concentration at probes 1-5 (left) and at probes 6-10 (right)
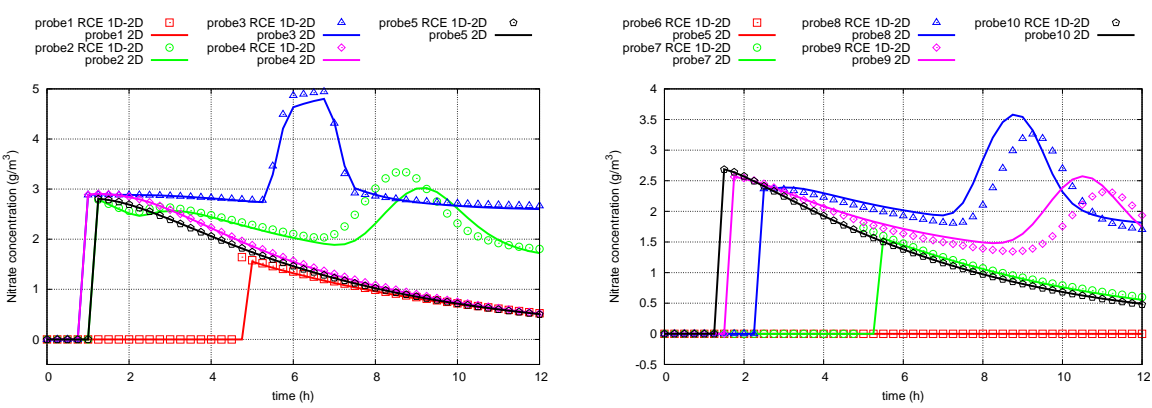

Fig. 15 Test case 1: Numerical solution of the time evolution of the nitrate concentration at probes 1-5 (left) and at probes 6-10 (right)

However, the probes are mostly well characterized and the overall behaviour is very similar to the $2 \mathrm{D}$ model, achieving good results in terms of accuracy. 
4.2 Test case 2 : Tiber river flooding with solute transport

The second test case consists of a realistic simulation of a five day flooding event in the Tiber river (Italy) combined with the transport of a passive solute, i.e., chlorine. A river reach of $6 \times 2 \mathrm{~km}$ close to the city of Rome is considered for a flooding event occurred in November 2005, in which the maximum peak discharge reached $1440 \mathrm{~m}^{3} / \mathrm{s}$. The measured hydrograph upstream the river reach is imposed as inflow discharge as well as a (not realistic) solute pulse which is also introduced at the inlet boundary (Figure 16, left).
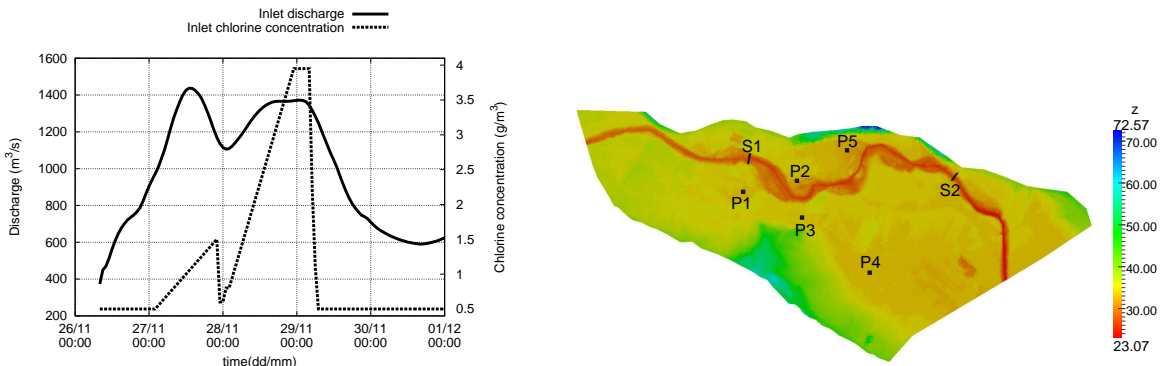

Fig. 16 Test case 2: upstream boundary condition (left) and topography and location of section and probes (right)

The outlet boundary condition is a gauging curve constructed from the measured discharge and water level at the end of the river reach. The Manning roughness coefficient is provided by the local administration distinguishing two zones: $n=0.035 \mathrm{~s} / \mathrm{m}^{1 / 3}$ inside the river and $n=0.0446 \mathrm{~s} / \mathrm{m}^{1 / 3}$. Field data is provided at two sections inside the main channel during all the episode. On the other hand, other probes or measured points are considered in order to compare both models. The spatial distribution of the cross sections as well as the probes are shown in (Figure 16, right). More information about the description of the test case can be found in (Morales-Hernández et al., In press).

For the simulation of this event, two numerical models are used: a fully 2D numerical model and the suggested coupled RCE 1D-2D model. A refined mesh only in the main channel is used as 2D reference, made of 26895 elements while a coarse mesh covers the floodplain in the fully $2 \mathrm{D}$ model as well as the $2 \mathrm{D}$ sub-domain in the $1 \mathrm{D}-2 \mathrm{D}$ coupled model. The detail of the two meshes used for the computation is displayed in Figure 17.

The recorded water elevations in sections S1 and S2 are compared, in Figure 18 , with the numerical results given by the fully $2 \mathrm{D}$ model and with the RCE 1D-2D model.

The numerical models can be also compared by using the information of the evolution in time registered at the probes $\mathrm{P} 1-\mathrm{P} 5$ by each numerical scheme 


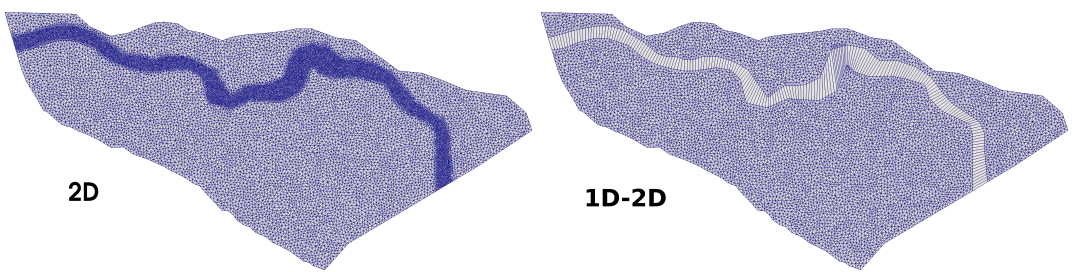

Fig. 17 Test case 2. Fully 2D mesh (left) and 1D-2D coupled mesh (right)
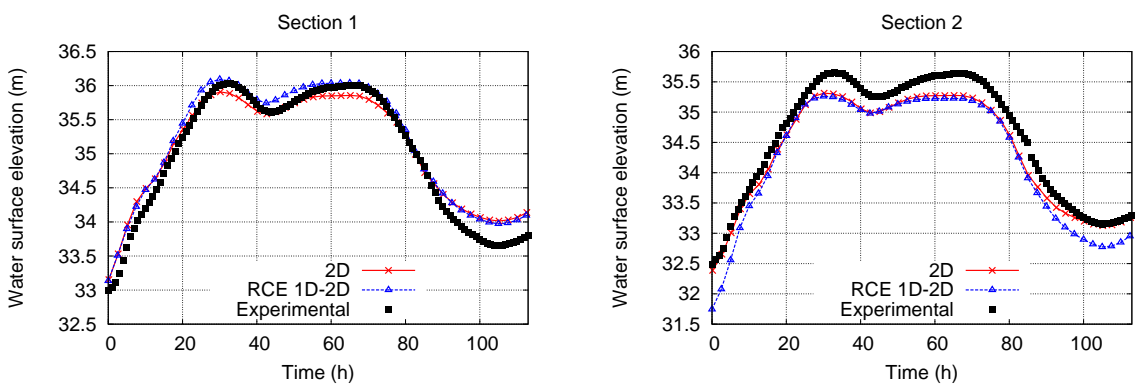

Fig. 18 Test case 2: Comparison between measured and computed data for sections S1 and S2

for the water depth (Figure 19, left) and for the chlorine concentration (Figure 19 , right).

As displayed in the previous figures, the RCE 1D-2D models and the fully $2 \mathrm{D}$ model generate almost the same results. Not only the maximum peaks in water surface elevation are fairly captured in comparison to the measured information but also the peak times are well reproduced and the flooding wave arrives almost at the same time in all the models. This behaviour is also observed at probes P1-P5 if compared to the 2D complete model for the water depth and for the solute evolution in time. In particular, all the numerical results underestimate the water peaks in section 2 hence it could be an effect of the downstream boundary condition, the Manning roughness coefficient (assumed constant along the main river) or even a bad representation of the bathymetry near this zone. Besides, the water depth is sometimes underestimated (mainly probe $\mathrm{P} 4$ ). With respect to the chlorine concentration, results are very similar in all the probes excluding $\mathrm{P} 1$, in which the solute is accumulated at the end of the event when using the RCE 1D-2D model. This can be due to the fact that probe 1 is located in a depression and the water depth remains disconnected until the end of the event.

This analysis is based on local measurements (sections and probes) along the domain. However, the differences can be estimated in terms of inundation maps generated by each numerical model. As an example, Figures 20 and 21 show two snapshots during the flood at times $\mathrm{t}=72 \mathrm{~h}$ and $\mathrm{t}=113 \mathrm{~h}$ (final state). Note that only the wet zones $(h>0.001 \mathrm{~m})$ are plotted. 

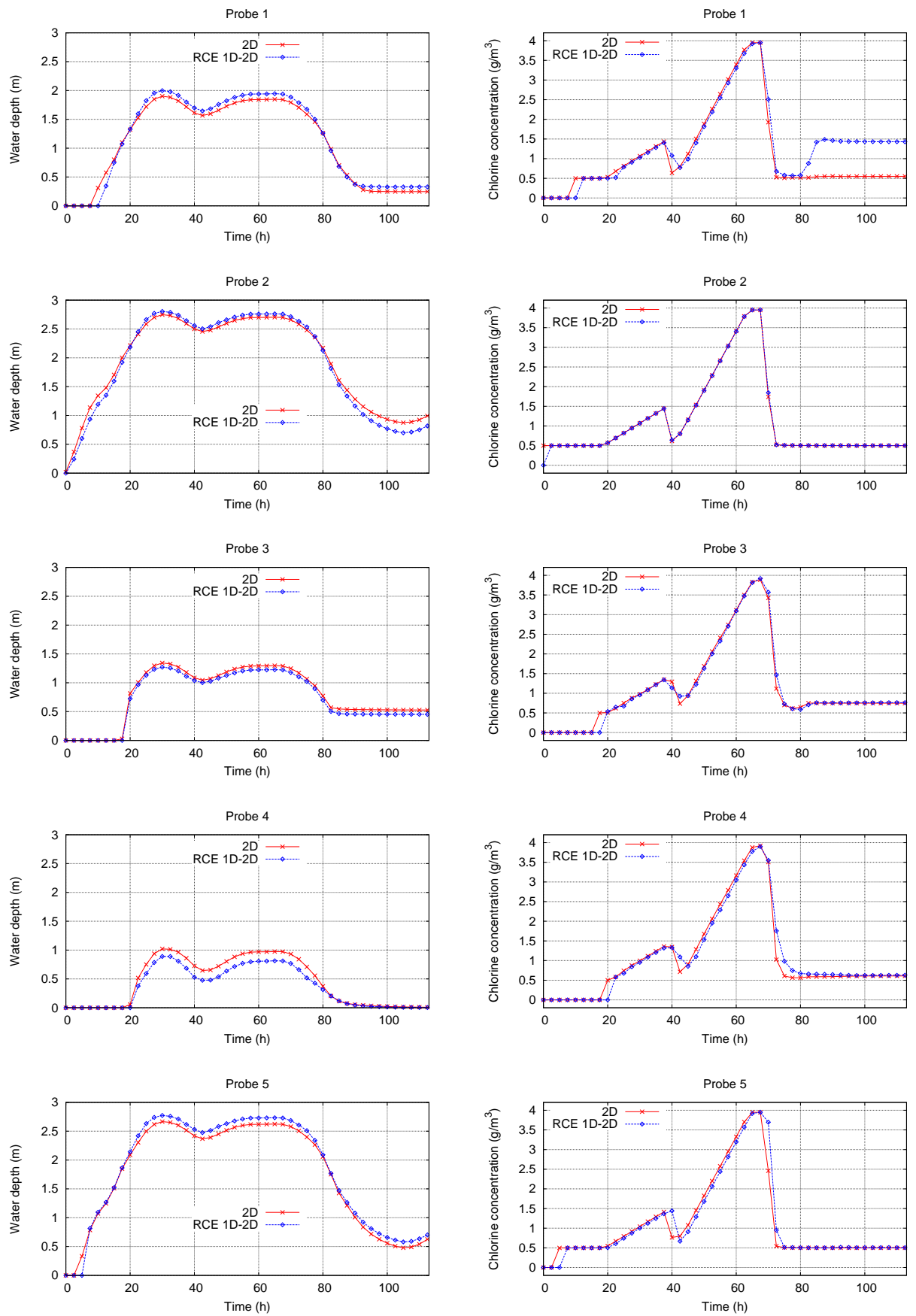

Fig. 19 Test case 2: Comparison of water depth (left) and chlorine concentration (right) among the different numerical models at probes P1-P5 

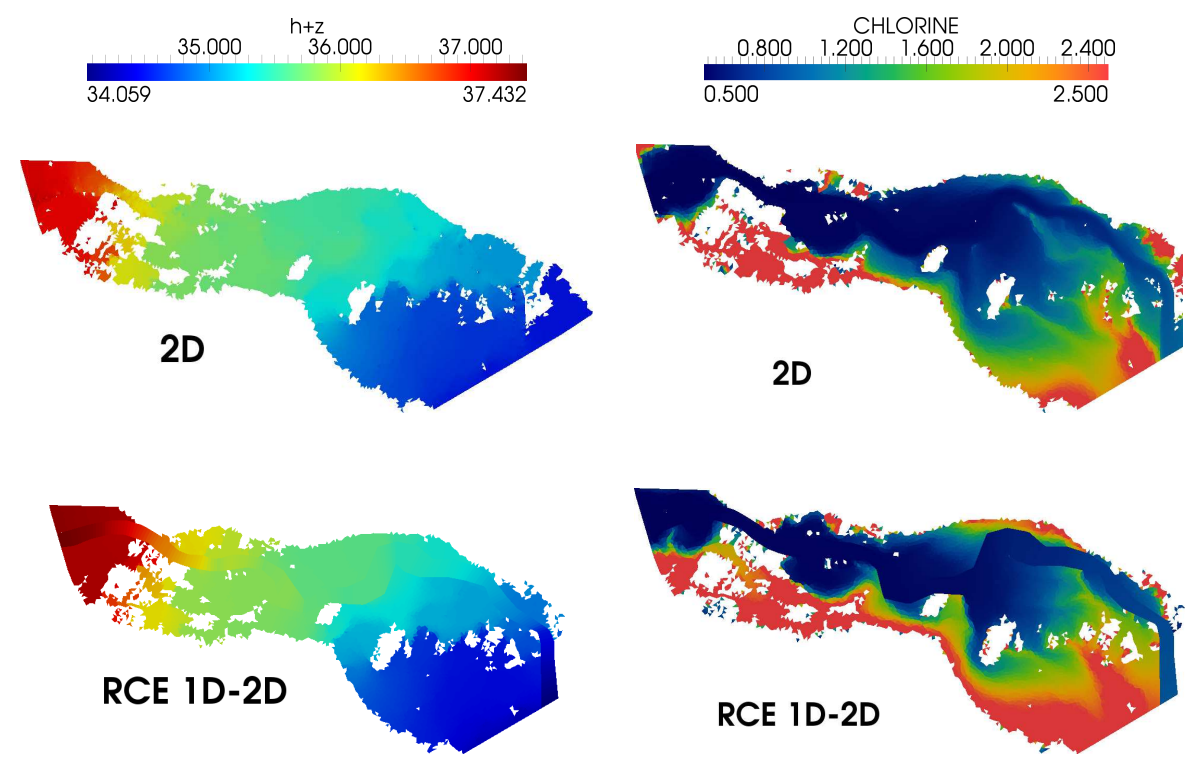

Fig. 20 Test case 2: Flooded area (left) and solute distribution (right) at time $t=72 \mathrm{~h}$, computed by the fully $2 \mathrm{D}$ model(upper) and RCE 1D-2D model (lower)

As can be observed, the 1D-2D coupled scheme is able to reproduce correctly the overall behaviour of the water surface elevation achieved by the $2 \mathrm{D}$ reference solution. However, at the right side of the end of the river reach, there is an area which is flooded by the $2 \mathrm{D}$ model and dried by the coupled model.

One main factor can be responsible for this: the 2D discretization in the river bathymetry. Firstly, this fact may increase the water level surface in the main river and, as a consequence, may overflow the right bank. Furthermore, it modifies the outlet boundary condition because the river capacity in the fully $2 \mathrm{D}$ model is different from coupled $1 \mathrm{D}-2 \mathrm{D}$ model, leading to a slightly different outlet boundary condition. Regarding the spatial distribution of the solute concentration, it can be concluded that is also well propagated by using the 1D-2D coupled model, although several regions are partially overestimated, closed to the boundaries.

\subsection{Computational time}

The level of accuracy of the proposed RCE 1D-2D model with respect to the 2D model has been already discussed. However, one of the main objectives of proposing a $1 \mathrm{D}-2 \mathrm{D}$ coupled model is related to the reduction in the computational time in comparison to the fully $2 \mathrm{D}$ model. They are two main factors: first of all, when using a 1D-2D coupled model, a considerable number of cells 

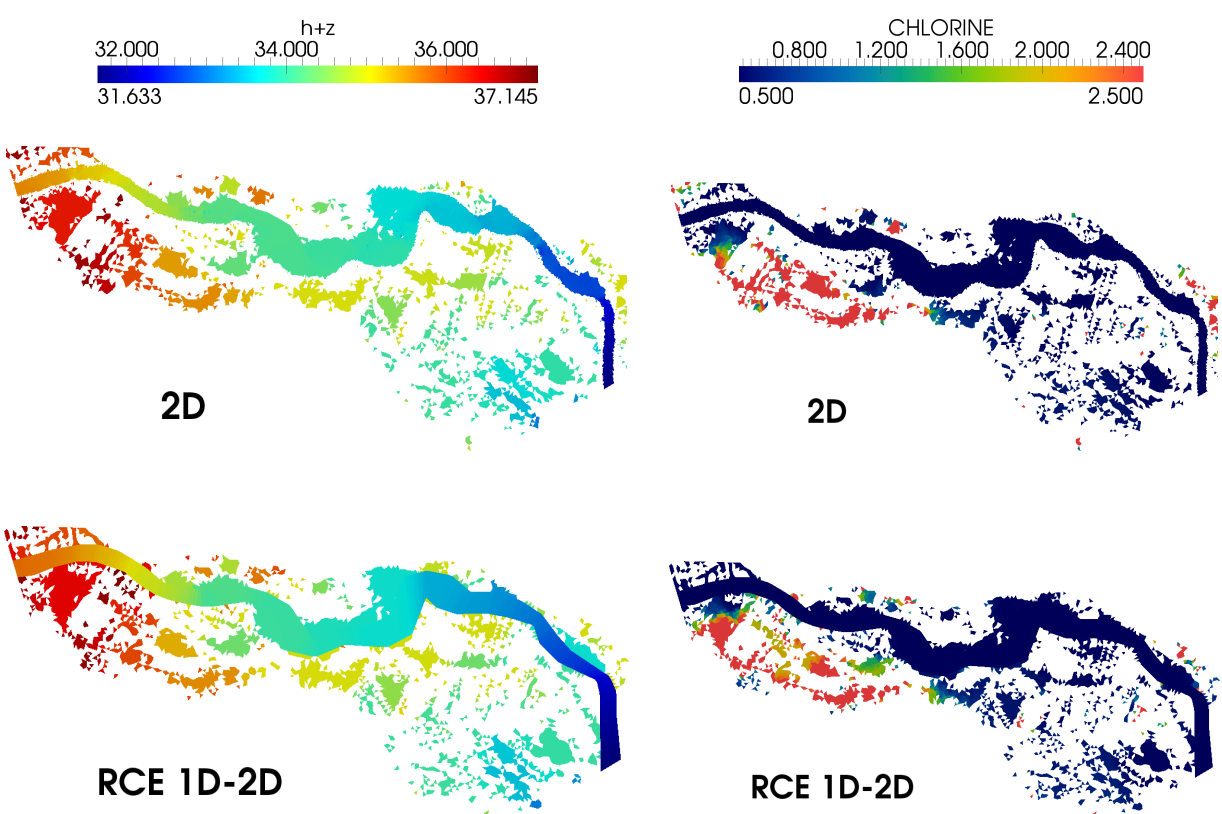

Fig. 21 Test case 6: Flooded area (left) and solute distribution (right) at time $\mathrm{t}=113 \mathrm{~h}$, computed by the fully 2D model(upper) and RCE 1D-2D model (lower)

are eliminated from the computation with respect to the $2 \mathrm{D}$ model. In particular, when simulating for instance the beginning of a flooding event and the river is canalized, the computational gain should be very large because only the $1 \mathrm{D}$ model is acting. On the other hand, far from eliminating cells, the cells which are removed reside in the main river hence they are small in size (in order to capture well the bathymetry of the river), they are always wet and they are usually responsible of the time step size. Therefore, the discard of these cells should be critical when examining the rapidity of both models.

The results for test cases 1 and 2 in terms of CPU time for both models are shown in Table 1. It is worth mentioning that the maximum triangle cell area constraint in the fully $2 \mathrm{D}$ model has been chosen equal from that of the $2 \mathrm{D}$ domain of the 1D-2D coupled model for each test case hence the uncertainty related with the choice of different computational cell sizes is eliminated. All the simulations were carried out in a Intel Core 2 Duo Quad Core Q9550 2.83 $\mathrm{GHz}$.

As expected, the results highlight a computational gain achieved by using the proposed RCE 1D-2D coupled model with speed-up's between $24 \mathrm{x}$ and $32 \mathrm{x}$. In particular, the performance of the test cases that involve the transport of substances decreases in comparison with the pure hydrodynamic test case. 


\begin{tabular}{|c|c|c|c|c|}
\hline \multirow[b]{2}{*}{ Test case } & & \multirow{2}{*}{$\begin{array}{l}\text { Fully } 2 \mathrm{D} \\
\text { Time }(s)\end{array}$} & \multicolumn{2}{|c|}{ RCE 1D-2D } \\
\hline & & & Time $(s)$ & Speed-up \\
\hline 1 & Steady & 66341 & 2120 & 31.29 \\
\hline & Solute transport & 39180 & 1496 & 26.19 \\
\hline 2 & & 120223 & 5006 & 24.01 \\
\hline
\end{tabular}

Table 1 CPU time consumed by the 2D model and the RCE 1D-2D model in each test case

\section{Conclusions}

A novel coupling strategy called Riemann Coupled Edges (RCE) between 1D and $2 \mathrm{D}$ shallow water models is proposed in this work. It is based on solving the Riemann Problem between the interfaces that separate the $1 \mathrm{D}$ and the $2 \mathrm{D}$ domains. Therefore, a smart meshing procedure is necessary in order to ensure a good geometric coupling between the models. Once the coupling zones are clearly established and an exact number of $2 \mathrm{D}$ cells are linked to a 1D cell, the quantities that come from the 1D model are bidimensionalized, that is, converted into $2 \mathrm{D}$ quantities considering the orientation of the coupling zone. Therefore, the RCE 1D-2D numerical scheme is reduced to solve the 1D-1D, the 2D-2D and the 1D-2D Riemann Problems that appear during the computation.

The proposed RCE 1D-2D coupled model is compared against a fully 2D model, not only on the basis of accuracy but also regarding the computational time spent by both models. It is demonstrated as accurate as the $2 \mathrm{D}$ model while the CPU time is decreased by a factor of 25 . Therefore, it is proved to be highly convenient when simulating this kind of phenomena.

\section{References}

Bermúdez, A. and Vázquez-Cendón, M.E, Upwind Methods for Hyperbolic Conservation Laws with Source Terms Computers Fluids, 23-8 (1994).

Bristeau, M. O. and Perthame, B., Transport of pollutant in shallow water using kinetic schemes, ESAIM Proceedings, Vol. 10, CEMRACS 1999, pp. 9-21 (2001).

Burguete, J. and García-Navarro, P., Efficient construction of high-resolution TVD conservative schemes for equations with source terms: application to shallow water flows, International Journal for Numerical Methods in Fluids, 37(2): 209-248. (2001)

Bladé E., Gomez-Valentín M., Dolz J., Quasi-two dimensional modelling of flood routing in rivers and flood plains by means of storage cells. Modelling of flood propagation over initially dry areas. ASCE, p. 156-170 (1994) 
Casulli, V. and Zanolli, P., High resolution methods for multidimensional advection-diffusion problems in free-surface hydrodynamics, Ocean Modelling, 10, 137-151. (2005)

Cunge J.A., Two dimensional modelling of floodplains. Unsteady flow in open channels, Water resources publications: 705-762, Fort Collins.(1975)

Fernández-Nieto E.D., Marin J., Monnier J., Coupling superposed 1D and 2D shallow water models: source terms in finite volume schemes, Computer \& Fluids 39: 1070-1082 (2010)

Finaud-Guyot P., Delenne C., Guinot V., Llovel C., 1D-2D coupling for river flow modelling, Comptes Rendus Mecanique 339: 226-234. (2011)

Gejadze I.Y., Monnier J., On a 2D zoom for the 1D shallow water model: coupling and data assimilation, Computer Methods in Applied Mechanics and Engineering, 196, 4628-4643. (2007)

Goutal, N, Parisot, M. Zaoui, F., A 2D reconstruction for the transverse coupling of shallow water models International Journal for Numerical Methods in Fluids 75 (11), 775-799 (2014).

Kuiry S.N., Sen D., Bates P.D., Coupled 1D- quasi 2D flood inundation model with unstructured grids, Journal of Hydraulic Engineering, 136(8):493-506 (2010)

Lin B., Wicks J.M., Falconer R.A. and Adams K., Integrating 1D and 2D hydrodynamic models for flood simulation, Proceedings of the Institution of Civil Engineers, Water Management, 159: 19-25. (2006)

Marin J., Monnier J., Superposition of local zoom models and simultaneous calibration for 1D-2D shallow water flows, Mathematics and Computers in Simulation, 80: 547-560, (2009)

Morales-Hernández M., García-Navarro P., Burguete J. and Brufau P., A conservative strategy to couple $1 \mathrm{D}$ and $2 \mathrm{D}$ models for shallow water flow simulation, Computers \& Fluids 81, 26-44. (2013)

Morales-Hernández M., Petaccia, G., Brufau P. and García-Navarro P., Conservative 1D-2D coupled numerical strategies applied to river flooding: the Tiber (Rome), Applied Mathematical Modelling, In press (2015)

Murillo, J., Burguete, J., Brufau, P. and García-Navarro, P., Coupling between shallow water and solute flow equations: analysis and management of source terms in 2D, International Journal for Numerical Methods in Fluids, 49 (3), 267-299 (2005)

Murillo, J. and García-Navarro, P. and Burguete, J., Analysis of a secondorder upwind method for the simulation of solute transport in $2 \mathrm{D}$ shallow water flow, International Journal for Numerical Methods in Fluids, 56 (6), 661-686 (2008)

Murillo, J. and García-Navarro, P., Weak solutions for partial differential equations with source terms: Application to the shallow water equations, J. Comput. Phys. 229 4327-4368. (2010)

Murillo, J. and García-Navarro, P., A Riemann solver for unsteady computation of $2 \mathrm{D}$ shallow flows with variable density, J. Comput. Phys. 231, 4775 $-4807 .(2012)$ 
Roe, P.L, Approximate Riemann solvers, parameter vectors and difference schemes, Journal of Computational Physics, 43, 357-372 (1981)

Vázquez-Cendón, M.E, Improved treatment of source terms in upwind schemes for SWE in channels with irregular geometries, Journal of Computational Physics 148 (2), 497-526 (1999)

Villanueva I., Wright N.G. , Linking Riemann and storage cell models for flood prediction, Proceedings of the Institution of Civil Engineers, Water Management, 159: 27-33. (2006)

\section{A Roe's average values}

The 1D Roe's linearized values used in this work are:

$$
\begin{gathered}
\tilde{\mathbf{e}}_{1}=\left(\begin{array}{c}
1 \\
\tilde{u}-\tilde{c}
\end{array}\right), \quad \tilde{\mathbf{e}}_{2}=\left(\begin{array}{c}
1 \\
\tilde{u}+\tilde{c}
\end{array}\right), \quad \tilde{\lambda}_{1}=\tilde{u}-\tilde{c}, \quad \tilde{\lambda}_{2}=\tilde{u}+\tilde{c}, \\
\tilde{u}_{i+1 / 2}=\frac{\sqrt{A_{i}} u_{i}+\sqrt{A_{i+1}} u_{i+1}}{\sqrt{A_{i}}+\sqrt{A_{i+1}}}, \quad \tilde{c}_{i+1 / 2}=\sqrt{g \frac{A_{i}+A_{i+1}}{B_{i}+B_{i+1}}} \\
\tilde{\alpha}_{1}=\frac{\tilde{\lambda}_{2} \delta A-\delta Q}{2 \tilde{c}}, \quad \tilde{\alpha}_{2}=\frac{-\tilde{\lambda}_{1} \delta A+\delta Q}{2 \tilde{c}}, \\
\tilde{\beta}_{1}=-\frac{1}{2 \tilde{c}}\left\{g \tilde{A}\left[\left(S_{0}-\tilde{S}_{f}\right) \Delta x-\delta h+\frac{1}{\tilde{B}} \delta A\right]\right\}, \quad \tilde{\beta}_{2}=-\tilde{\beta}_{1},
\end{gathered}
$$

where the tilde variables represent an average state at each edge. The average states $\tilde{A}, \tilde{B}$ and $\tilde{S}_{f}$ can be easily defined as arithmetic mean (Burguete and García-Navarro, 2001) in the conventional formulation.

For the $2 \mathrm{D}$ numerical scheme, the average eigenvectors and eigenvalues are given by:

$$
\begin{gathered}
\tilde{\mathbf{e}}_{1}=\left(\begin{array}{c}
1 \\
\tilde{u}-\tilde{c} n_{x} \\
\tilde{v}-\tilde{c} n_{y}
\end{array}\right), \quad \tilde{\mathbf{e}}_{2}=\left(\begin{array}{c}
0 \\
-\tilde{c} n_{y} \\
\tilde{c} n_{x}
\end{array}\right), \quad \tilde{\mathbf{e}}_{3}=\left(\begin{array}{c}
1 \\
\tilde{u}+\tilde{c} n_{x} \\
\tilde{v}+\tilde{c} n_{y}
\end{array}\right), \\
\lambda_{1}=\tilde{\mathbf{u}} \cdot \mathbf{n}-\tilde{c}, \quad \lambda_{2}=\tilde{\mathbf{u}} \cdot \mathbf{n}, \quad \lambda_{3}=\tilde{\mathbf{u}} \cdot \mathbf{n}+\tilde{c} \\
\tilde{u}_{k}=\frac{\sqrt{h_{i}} u_{i}+\sqrt{h_{j}} u_{j}}{\sqrt{h_{i}}+\sqrt{h_{j}}}, \quad \tilde{v}_{k}=\frac{\sqrt{h_{i}} v_{i}+\sqrt{h_{j}} v_{j}}{\sqrt{h_{i}}+\sqrt{h_{j}}}, \quad \tilde{c}_{k}=\sqrt{g \frac{h_{i}+h_{j}}{2}}
\end{gathered}
$$

while the wave and source strengths are expressed

$$
\begin{gathered}
\left.\left.\tilde{\alpha}_{1}=\frac{\delta h}{2}-\frac{1}{2 \tilde{c}}(\delta \mathbf{q} \cdot \mathbf{n}-\tilde{\mathbf{u}} \cdot \mathbf{n} \delta h), \quad \tilde{\alpha}_{2}=\frac{1}{\tilde{c}}\left[\delta q_{y}-\tilde{v} \delta h\right) n_{x}-\left(\delta q_{x}-\tilde{u} \delta h\right) n_{y}\right)\right], \\
\tilde{\alpha}_{3}=\frac{\delta h}{2}+\frac{1}{2 \tilde{c}}(\delta \mathbf{q} \cdot \mathbf{n}-\tilde{\mathbf{u}} \cdot \mathbf{n} \delta h), \\
\tilde{\beta}_{1}=\frac{g \tilde{h}}{2 c}\left(\delta z+S_{f, \mathbf{n}}\right), \quad \tilde{\beta}_{2}=0, \quad \tilde{\beta}_{3}=-\tilde{\beta}_{1}
\end{gathered}
$$

where $\tilde{\mathbf{u}} \cdot \mathbf{n}=\tilde{u} n_{x}+\tilde{v} n_{y}, \delta \mathbf{q} \cdot \mathbf{n}=\delta q_{x} n_{x}+\delta q_{y} n_{y}$ and the averages states at each wall $k$ are represented with the tilde variables. $S_{f, \mathbf{n}}$ accounts for the discretized and projected friction term, which is expressed as follows (Murillo and García-Navarro, 2010): 


$$
S_{f, \mathbf{n}}=\frac{\bar{n}^{2} \tilde{\mathbf{u n}} \min \left(\left|u_{i}\right|,\left|u_{j}\right|\right)}{\max \left(h_{i}, h_{j}\right)^{4 / 3}} d_{n}
$$

where $\bar{n}=\frac{1}{2}\left(n_{i}+n_{j}\right)$ is the averaged Manning's coefficient and $d_{n}$ is the normal distance between cell centers. 